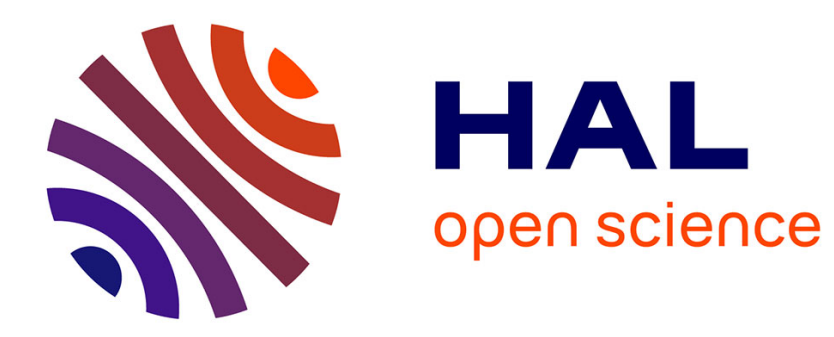

\title{
Bridge function for the dipolar fluid from simulation
}

Joël Puibasset, Luc Belloni

\section{To cite this version:}

Joël Puibasset, Luc Belloni. Bridge function for the dipolar fluid from simulation. Journal of Chemical Physics, 2012, 136. hal-02110035

\section{HAL Id: hal-02110035 \\ https://hal.science/hal-02110035}

Submitted on 26 Nov 2019

HAL is a multi-disciplinary open access archive for the deposit and dissemination of scientific research documents, whether they are published or not. The documents may come from teaching and research institutions in France or abroad, or from public or private research centers.
L'archive ouverte pluridisciplinaire HAL, est destinée au dépôt et à la diffusion de documents scientifiques de niveau recherche, publiés ou non, émanant des établissements d'enseignement et de recherche français ou étrangers, des laboratoires publics ou privés. 


\title{
Bridge function for the dipolar fluid from simulation
}

Joël Puibasset ${ }^{1}$ and Luc Belloni ${ }^{2}$

${ }^{1}$ CRMD, CNRS, Université d'Orléans, $1 B$ rue de la Férollerie, 45071-Orléans Cedex, France

${ }^{2}$ CEA/Saclay, SIS2M, LIONS, 91191-Gif-sur-Yvette Cedex, France

\begin{abstract}
The exact bridge function of the Lennard-Jones dipolar (Stockmayer) fluid is extracted from Monte Carlo simulation data. The projections $g^{m n l}(r)$ onto rotational invariants of the nonspherically symmetric pair distribution function $g(r, \Omega)$ are accumulated during simulation. Making intensive use of anisotropic integral equation techniques, the molecular OrnsteinZernike equation is then inverted in order to derive the direct correlation function $c^{m n l}(r)$, the cavity function $y^{m n l}(r)$, the negative excess potential of mean force $\left.\ln y\right|^{m n l}(r)$ and the bridge function $b^{m n l}(r)$ projections. $b(r, \Omega)$ presents strong, non-universal anisotropies at high dipolar coupling. This simulation data analysis may serve as reference and guide for approximated bridge function theories of dipolar fluids and is a valuable step towards the case of more refined, nonlinear water-like geometries.
\end{abstract}

puibasset@cnrs-orleans.fr ; luc.belloni@cea.fr 


\section{INTRODUCTION}

The basic problem in statistical physics of liquids consists to derive the pair distribution function $(p d f) g$ from the pair potential $v$. A formal exact relation which involves total $h=g-1$ and direct $c$ correlation functions reads $\mathbf{1}$ :

$$
g(12)=\exp (-\beta v(12)) y(12)=\exp (-\beta v(12)+\ln y(12))=\exp (-\beta v(12)+h(12)-c(12)+b(12))
$$

where $y$ is usually called the cavity function, $b$ is the bridge function and $\beta=1 / k T$ the inverse thermal energy. For spherically symmetric fluids, each function depends on the distance $r$ between particles only, $(12) \equiv\left(r_{12}\right) \equiv(r)$, while for anisotropic fluids as the dipolar system under the present study, it depends on the (relative) orientations of the two particles $\Omega_{1}, \Omega_{2}$ and the vector joining them $\hat{r} \equiv \hat{r}_{12}$ as well, $(12) \equiv(r, \Omega)$. The Ornstein-Zernike (OZ) equation gives a second exact relation between $h$ and $c$ correlation functions $\mathbf{1}$ :

$$
\gamma(12)=h(12)-c(12)=\rho \int h(13) c(32) d 3
$$

where $\rho$ is the number concentration. The negative excess potential of mean force $\ln y(12) \equiv \chi(12)+b(12)$ is thus the sum of two contributions: the first one, $\gamma$, can be easily related through the OZ convolution product (2) (algebraic product in Fourier space) to the pair distribution function while the second one, $b$, despite existing formal diagrammatic or functional exact expressions, is usually unknown. The ultimate goal in such basic statistical mechanical problem is to make a reasonable (if not the best possible) assumption for the bridge function $b$ and to solve the corresponding approximated closure or integral equation (1) coupled with the OZ equation (2) through an iterative procedure which makes an intensive use of numerical Fourier transforms (FT). A comparison of the derived $p d f$ with exact reference data provided by numerical simulation reveals a posteriori the quality of the assumed $b$.

The simplest choice consists to neglect the bridge function, $b(12)=0$; that defines the very popular HNC integral equation. This approximation has been widely used with clear success for various systems since the 1960 's, from neutral atomic liquids ${ }^{2}$ to molten salts ${ }^{3}$ or electrolytes ${ }^{4}$, from asymmetrical charged colloids ${ }^{5}$ to molecular polar solvents ${ }^{6} \ldots$ Then, approximated bridge functions have been proposed following different directions. For 40 years, the literature is full of theories based for instance on ad-hoc universality hypothesis borrowing bridge from a reference hard-sphere fluid ${ }^{7}$, on diagrammatic expansions ${ }^{8}$, on phenomenological mixed closures ${ }^{9} \mathbf{1 0}$, on density functional approach ${ }^{\mathbf{1 1}}$, on three-body distribution functions ${ }^{\mathbf{1 2}}$...

Rather to compare and test theories with exact simulation data at the $p d f$ level, it is also interesting to do it at the bridge function level itself. For that purpose, the exact bridge function must be extracted from given $g(r)$ simulation values (inverse problem). In principle, 
the extraction is straightforward, at least for spherically symmetric potentials: the $\gamma(r)$ function is derived from the numerical $g(r)$ data through the OZ equation (2) and two (one direct, one inverse) FTs. Then, (1) gives the desired $b$ function via the simple identity $b=\ln g+\beta v-\gamma$. In practice, the situation is not so simple. Beyond the problem of statistical noise intrinsically always present in any numerical simulation, the "measured" profile $g(r)$ is neither complete nor exact, due to the finite size $L$ of the simulation cell. First, information is available in a limited $r$ range only $\left(r<r_{\max } \approx L / 2\right)$. For most standard simulations, that cut-off is not large compared to the correlation range, especially in dense systems and/or at high electrostatic coupling, and the missing tail of $g(r)$ cannot be safely ignored. That opens to the general problem of extending simulation data beyond $r_{\text {max }}$ with some extrapolation scheme, based for instance on approximate bare integral equations ${ }^{\mathbf{1 3}}$ (in that spirit, the extraction of bridge function starts by facing the same difficulties than in the calculation of the so-called Kirkwood-Buff integrals of the $p d f$ which are related to thermodynamic derivative properties; this domain is the subject of recent intense studies, see for instance ${ }^{\mathbf{1 4}}$ ). Secondly, even the available information below $r_{\max }$ is corrupted by systematic corrections. Despite these numerical limitations and difficulties, exact bridge functions are now known in the literature for a limited number of simple spherical potentials like the Lennard-Jones (LJ) ${ }^{\mathbf{1 5}}$, the soft sphere ${ }^{\mathbf{1 6}}$ or the hard-sphere ${ }^{\mathbf{1 7}}$ ones, which can serve as benchmarks for approximated theories.

On the other hand, little has been done in that respect for non-spherical particles. The reason is certainly due to the numerical difficulty to deal with correlation functions that depend on separation $r$ and orientations $\Omega$. As usual in the treatment of anisotropic particles, rather to manipulate the complete $g(r, \Omega)$ as explicit function of the different Euler angles, it is more fruitful to project it onto a basis of angular functions and to play with the projections that depend on the separation $r$ only. Following Blum's notation and normalization ${ }^{18}{ }^{19}$ :

$$
g(r, \Omega) \equiv g\left(r, \Omega_{1}, \Omega_{2}, \hat{r}\right)=\sum_{m, n, l, \mu, v} g_{\mu \nu}^{m n l}(r) \Phi_{\mu \nu}^{m n l}\left(\Omega_{1}, \Omega_{2}, \hat{r}\right)
$$

with

$$
\Phi_{\mu \nu}^{m n l}\left(\Omega_{1}, \Omega_{2}, \hat{r}\right)=\sqrt{(2 m+1)(2 n+1)} \sum_{\mu^{\prime} \nu^{\prime}, \lambda^{\prime}}\left(\begin{array}{ccc}
m & n & l \\
\mu^{\prime} & v^{\prime} & \lambda^{\prime}
\end{array}\right) R_{\mu^{\prime} \mu}^{m}\left(\Omega_{1}\right) R_{v^{\prime} v}^{n}\left(\Omega_{2}\right) R_{\lambda^{\prime} 0}^{l}(\hat{r})
$$

Similar expansions (3) hold for each correlation function. The coefficients $\left(\begin{array}{ccc}m & n & l \\ \mu^{\prime} & v^{\prime} & \lambda^{\prime}\end{array}\right)$ are the usual 3-j-symbols. The $R_{\mu^{\prime} \mu}^{m}(\Omega)$ are Wigner generalized spherical harmonics (definition and notation from Messiah ${ }^{20}$ ). The $\Phi_{\mu \nu}^{m n l}$ rotational invariants (independent of the reference frame) form an orthogonal basis. They depend on the relative orientation of the two molecules and of the vector joining them (five Euler angles) and are characterized by five indices $m, n, l$, $\mu, v$. For linear particles (axis $\hat{\mu}$ ) as in the present dipolar fluid study, three Euler angles are sufficient, $m+n+l$ is even and $\mu=v=0$. In the following, although much of the analysis will be valid for any anisotropy, the indices $\mu, v$ will be dropped to simplify the notation. The first 
terms $\quad$ are $\quad \Phi^{000}=1, \Phi^{110}=-\sqrt{3} \hat{\mu}_{1} \hat{\mu}_{2}, \Phi^{112}=\sqrt{3 / 10}\left(3\left(\hat{\mu}_{1} \hat{r}\right)\left(\hat{\mu}_{2} \hat{r}\right)-\hat{\mu}_{1} \hat{\mu}_{2}\right), \ldots \quad$ (note $\quad$ the coefficients $-\sqrt{3}$ and $\sqrt{3} / 10$ instead of the more standard value 1 for the definition of the $\Phi^{110}$ and $\Phi^{112}$ invariants, respectively, which results from Blum's normalization; that induces the inverse coefficients in the corresponding projections). The coefficients $g^{m n l}(r)$ are derived from the complete function by angular projection:

$$
g^{m n l}(r)=\iiint g(r, \Omega) \Phi^{m n l} *(\Omega) d \Omega / \iiint\left|\Phi^{m n l}\right|^{2}(\Omega) d \Omega \equiv(2 l+1) \iiint g(r, \Omega) \Phi^{m n l}(\Omega) d \Omega
$$

In short notation, if $\Phi_{\alpha}$ stands for $\Phi^{m n l}$, expansion and projection read:

$$
\begin{aligned}
& g(r, \Omega)=\sum_{\alpha} g_{\alpha}(r) \Phi_{\alpha}(\Omega) \\
& g_{\alpha}(r)=\left\langle g(r, \Omega) \Phi_{\alpha}{ }^{*}(\Omega)\right\rangle
\end{aligned}
$$

where the brackets in (7) represents a (normalized) triple angular integral. The first projection $g_{1}=g^{000}$ represents the center of mass-center of mass $p d f$ (averaged over all orientations).

In principle, the basis is infinite. In practice, it is assumed and verified that a limited number $\alpha_{\max }$ of projections, characterized by $m, n \leq n_{\max }$, is sufficient, at least for the excess potential of mean force lny, to quantitatively capture the angular dependence of the correlations.

The first projections $b^{m n l}(r)$ of the bridge function have been recently extracted from Monte Carlo (MC) projections $g^{m n l}(r)$ for hard spheroid ${ }^{21}$ and hard platelet ${ }^{22}$ fluids. As for the case of spherical interactions, the MC data available at $r<r_{\max }$ have been first completed with the MSA approximation (here $c(r)=0$ ) at larger distances, $r>r_{\max }$. Then, the projections $c^{m n l}(r)$ of the direct correlation function have been derived by inverting the OZ equation. The key final point is to calculate the projections of $\ln y$ (the exponent) from those of $g$ (the exponential). That difficult step requires careful methodologies based on the techniques developed for solving integral equations in the case of anisotropic particles ${ }^{6}$.

The purpose of the present study is to derive the exact bridge projections $b^{m n l}(r)$ from $p d f$ $g^{m n l}(r)$ data provided by MC simulation for the LJ dipolar (or Stockmayer) fluid. To the author's knowledge, no such information is available in the literature. Only the direct correlation function has been derived from MC data (completed again with MSA approximation at large distances), not for extracting the bridge but for feeding the density functional theory to describe solvation properties of molecular solutes ${ }^{\mathbf{2 3}}$. The pair potential is the sum of spherical LJ and highly anisotropic dipole-dipole contributions:

$$
v(r, \Omega)=4 \varepsilon\left(\left(\frac{\sigma}{r}\right)^{12}-\left(\frac{\sigma}{r}\right)^{6}\right)-\frac{\mu^{2}}{4 \pi \varepsilon_{0} r^{3}}\left(3\left(\hat{\mu}_{1} \cdot \hat{r}\right)\left(\hat{\mu}_{2} \cdot \hat{r}\right)-\left(\hat{\mu}_{1} \cdot \hat{\mu}_{2}\right)\right)
$$

$\sigma$ and $\varepsilon$ are the usual size and energy LJ parameters, $\mu$ is the dipole moment. The LJ term contributes to $v^{000}$ while the dipole-dipole potential reads $v^{112}(r)=-\sqrt{10 / 3} \frac{\mu^{2}}{4 \pi \varepsilon_{0} r^{3}}$. For such 
purely dipolar anisotropy, $\left(\vec{\mu}_{1}, \vec{\mu}_{2}\right)$ and $\left(-\vec{\mu}_{1},-\vec{\mu}_{2}\right)$ configurations are equivalent and the projections $m, n, l$ are restricted to even $l$ (and so even $m+n$ ).

All systems investigated in this paper correspond to $T^{*}=k T / \varepsilon=2, \rho^{*}=\rho \sigma^{3}=0.8$ and three dipolar coupling $\mu^{* 2} \equiv \frac{\mu^{2}}{4 \pi \varepsilon_{0} \sigma^{3} k T}=1.5,2.25$ and 3. The LJ potential is truncated, shifted at $r_{c}=2.5 \sigma$. These systems correspond to dense polar solvents of increasing dielectric constants and are characterized by strong short and long range correlations.

The MC simulation is described in section II; beside the standard construction of the $p d f$ from the neighbor configurations inside the cell, a special technique based on the Henderson method ${ }^{\mathbf{2 4}}$ is needed to get information on the cavity function inside the soft core. Section III presents the extraction of the bridge function by solving the inverse problem and making use of all the technical machinery developed for the resolution of integral equations in the case of anisotropic potentials. The main difficulty is to relate projections of the exponent and of the exponential in (1), or $y^{m n l}$ and $\ln y^{m n l}$ (which must be understood in the following as $(\ln y)^{m n l}$ and not be confused with $\ln \left(y^{m n l}\right)$ ). The results in terms of the $p d f$, the direct correlation function, the cavity function, the excess potential of mean force and the bridge function are finally presented and discussed in section IV for the different dipolar strengths.

\section{Monte Carlo simulation}

Since the simulation data are designated to be the source in the inverse problem, the required precision is much more restrictive than in a standard study which usually needs good looking $p d f$ curves. That not only means large number $N$ of particles and long simulations, but also correct control of all possible bias.

$N=100$ to 800 dipolar particles are put in a cubic cell of edge $L=5 \sigma$ to $10 \sigma\left(\rho=N / L^{3}\right)$ with periodic boundary conditions ( $N, V, T$ canonical ensemble). The long range dipole-dipole interaction is dealt with the standard Ewald technique ${ }^{25}$. Beside the exact treatment of electrostatics in periodic systems, the advantage of this technique (compared to reaction field methods for instance) is that the possible artifacts arising in correlation functions due to periodic boundary conditions can be exactly evaluated (and possibly removed). The Ewald energy classically contains two sums, one in the $r$ space with $\operatorname{erfc}\left(\kappa\left|\boldsymbol{r}_{i j}+\boldsymbol{n} L\right|\right)$ typical terms, the other one expressed in the reciprocal $k$ space with $\exp \left(-k^{2} / 4 \kappa^{2}\right)=\exp \left(-(\pi|\boldsymbol{m}| / \kappa L)^{2}\right)$ factors $(\boldsymbol{n}, \boldsymbol{m}$ are vectors of integer components). The former is spherically cut at $r=L / 2$ (so, only the minimum image distance, $n=0$, is retained). The screening constant $\kappa$ is chosen such that the factor $\kappa r$ at the cut is equal to $s=4$, so $\kappa L=8$. In the latter truncated sum, the largest $\boldsymbol{m}$ vector is similarly chosen such that $\pi m / \kappa L=s=4$, so $m_{\max }=4 \kappa L / \pi=10$ (647 independent $\boldsymbol{m}$ vectors). With this choice, the relative precision in the dipolar energy is better than $10^{-6}$, each sum contributing on equal level to the uncertainty. Note that this precision is much better than the standard ones given in the literature which correspond to lower values $(\approx 3)$ for the $s$ 
parameter. With this strong constraint, we are sure to avoid any bias related to a limited precision in the Ewald summation ${ }^{\mathbf{2 6}}$.

Another key quantity in the Ewald technique is the dielectric constant $\varepsilon^{\prime}$ of the external medium surrounding the central box and its replica, which explicitly appears in the energy ${ }^{25}$. It is well known that choosing two different $\varepsilon^{\prime}$ values leads to two $p d f$ s which differ by $1 / N$ leading corrections ${ }^{27}$. In particular, the $h^{110}(r)$ projection presents a non-zero constant asymptote equal to:

$$
h_{\varepsilon^{\prime}}^{110}(\text { asymptote })=-\frac{2}{3 \sqrt{3} y} \frac{\left(\varepsilon^{\prime}-\varepsilon\right)(\varepsilon-1)^{2}}{\varepsilon\left(2 \varepsilon^{\prime}+\varepsilon\right)} \frac{1}{N}
$$

where $y=4 \pi \rho^{*} \mu^{* 2} / 9$ and $\varepsilon$ is the dielectric constant of the system itself, which can be derived from a relation which depends explicitly on $\varepsilon^{25}$ :

$$
\frac{(\varepsilon-1)\left(2 \varepsilon^{\prime}+1\right)}{2 \varepsilon^{\prime}+\varepsilon}=\frac{4 \pi}{3} \frac{\left\langle M^{2}\right\rangle}{4 \pi \varepsilon_{0} L^{3} k T}=3 y g
$$

The usually known $g$-factor, $g=\left\langle M^{2}>/ N \mu^{2}\right.$, is given by the orientational order fluctuations of the box ( $M$ is the total moment of zero theoretical average $\langle M\rangle$ ) or by the integral of $h^{110}$ :

$$
g=1-\frac{\rho}{\sqrt{3}} \int h^{110}(r) 4 \pi r^{2} d r
$$

The asymptote (9) guarantees that $\varepsilon$ is (fortunately) independent of the boundary conditions $\varepsilon^{\prime}$ in the thermodynamical limit ${ }^{27}$. We have performed various MC simulations at different $N$ and $\varepsilon$. The $1 / N$ detailed analysis of the observed differences will be presented in a future paper ${ }^{28}$. For the present inverse problem and bridge extraction, it is important to start from simulation $p d f$ s which represent infinite bulk volumes as close as possible. In particular, it is impossible to feed the inverse problem with non-zero $h^{110}$ asymptote! Indeed, even if that asymptote behaves as $O(1 / N)$ and could be in principle arbitrary reduced by choosing large $N$, it leads to a $O(1)$ term when integrated over the box volume (or over an important fraction of it). So, without precaution, the FT of the $\mathrm{MC} h^{110}(r)$ function would present spurious behavior at low scattering vector $q$. So, it is at least necessary to subtract the known asymptote (9) from the accumulated $h^{110}(r)$ function, or better, to choose $\varepsilon^{\prime}$ as close as possible to $\varepsilon$. That guarantees the absence of dielectric discontinuity between the system (central box + replica) and the external medium and kills the $1 / N$ corrections (asymptote as well as more or less controlled short-range corrections). In practice, we first perform a short simulation with $N=100$ to derive an estimate of $\varepsilon$. Then, the long simulations at larger $N$ are produced with $\varepsilon$ equal to that estimate. At the end, we verify that the residual asymptote coming from a small difference between the accurate $\varepsilon$ and $\varepsilon$ can be safely ignored.

Each trial displacement is made of a local translation inside a cube (of size $\approx 0.1-0.2 \sigma$ ) and of a rotation of the dipole axis inside a cone (of angle $\approx 40-60^{\circ}$ ) and is accepted according to the Metropolis method. After equilibration, the projections $g^{m n l}(r)$ are constructed from the 
relative positions and orientations of the particles in the box. For each retained configuration, the minimum image separations $r_{i j}$ of all pairs $i<j$ of dipoles are sorted into a histogram of width $\delta r(\approx \sigma / 80)$. The bin $k$ corresponds to the distance interval $\mathrm{I}_{k}=\left[r_{k-1}, r_{k}\right]$ with $r_{k}=k \delta r$, and to the volume of the spherical cap $V_{k}=4 \pi / 3\left(r_{k}^{3}-r_{k-1}{ }^{3}\right)$. So, following the definition (5), the projections are constructed according to:

$$
g^{m n l}(\operatorname{bin} k)=\frac{2 l+1}{V_{k} N^{2} / 2} \sum_{\substack{i<j \\ r_{i j} \in I_{k}}} \Phi^{m n l}{ }^{*}\left(\hat{\mu}_{i}, \hat{\mu}_{j}, \hat{r}_{i j}\right)
$$

Note the correct normalization factor $N^{2} / 2$ (and not $N(N-1) / 2$ ). Due to the finiteness of the width $\delta r$, that information in the bin $k$ measures some average of $g$ over the interval $\mathrm{I}_{k}$ and is usually attributed to the mean separation $\left(r_{k-1}+r_{k}\right) / 2$. In order to reduce the convolution effect, we go a little bit further: the construction in histograms measures in fact numbers of neighbors, so calculates directly not $g$, but its primitive $G(r)=\int_{0}^{r} g(s) s^{2} d s$ at each $r=r_{k}$. So, we make a spline cubic interpolation of the MC data $G\left(r_{k}\right)$, take the analytical derivative and divide by $r^{2}$ to construct refined values of $g\left(r_{k}\right)$ for each projection. That procedure improves the final function $\ln y^{m n l}$ especially at the bottom of the first peak in $g$, near $r=\sigma$, where $v$ and $g$ vary rapidly.

It is important to remember that the simulated fluid is not perfectly isotropic due to the periodic boundary conditions and the correlation $(i j)$ depends not only on the relative orientations of $\hat{\mu}_{i}, \hat{\mu}_{j}$ and $\hat{r}_{i j}$ but also weakly on the absolute orientation of the ensemble with respect to the axes of the box. The expansion (3) in rotational invariants is recovered only by averaging over that absolute orientation, as in (12).

In the present study, $\alpha_{\max , \mathrm{MC}}=7$ projections are usually accumulated, which correspond to $n_{\max , \mathrm{MC}}=2$. For the highest dipole, we have refined the analysis by accumulating 13 projections $\left(n_{\max , \mathrm{MC}}=3\right)$. For each pair, the spherical harmonics $\Phi^{110}, \Phi^{112}$ and $\Phi^{022}$ (or $\Phi^{202}$ ) are calculated from the scalar products between the three unit vectors. The remaining harmonics, $\Phi^{221}$ (and $\Phi^{131}, \Phi^{331}$ ) are deduced from products of the previous ones. No manipulation of trigonometric functions is required. At the end, the MC simulation provides 7 or 13 projections of the $p d f$ in the limited range $r<r_{\max , \mathrm{MC}}=L / 2$ (we did not try to extent the $r$ regime by exploring the corners of the box because these data are sensitive to bias ${ }^{29}$ ). Beside the statistical noise related to the finite length of the simulation, the so constructed data are known to be subjected to systematic finite-size corrections, the explicit corrections due to the absence of fluctuations in the number of particles within the canonical simulation ${ }^{\mathbf{3 0}}$ and the implicit corrections due to the coupling between the environment around a given particle and that around its images in the neighboring cells ${ }^{31}$. The detailed analysis of these different types of corrections for dipolar fluids will be given in the future paper ${ }^{28}$. For the present study, it is necessary and sufficient to account for the finite-size explicit correction in the long distance asymptote of the $p d f^{\mathbf{3 0}}$ : 


$$
\lim _{\text {larger } r} g_{N}(r, \Omega)=\lim _{\text {large } r} g_{N}^{000}(r)=1-\frac{\chi}{N}
$$

where $\chi=\left(\frac{\partial \rho}{\partial \beta P}\right)_{T}$ is the normalized isothermal compressibility ( $P$ is the pressure) which is related to the center of mass structure factor $S^{000}(q)$ at zero $q$, $\chi=S^{000}(0)=1+\rho \int_{0}^{\infty}\left(g^{000}-1\right) 4 \pi r^{2} d r$. The departure from the asymptote 1 in (13) simply means that the number of neighbors available to the far field environment around a given particle is $N$ minus 1 (the central particle itself) and minus the excess particles locally adsorbed or desorbed around it (given by the integral of $g-1$ ), so is $N-\chi$. In grand-canonical conditions, this finite-size correction exactly vanishes due to the exchange of particles with the reservoir. As before, this $O(1 / N)$ asymptotic correction, which may be in the $10^{-3}-10^{-4}$ range, leads without precaution to a $O(1)$ spurious effect in $S^{000}(q)$ at low $q$. So, all MC projections have been multiplied by the factor $1 /(1-\chi / N) \approx 1+\chi / N$ before use in order to recover the bulk asymptote 1 . For the very same reason, the value of $\chi$ needed in this renormalization cannot be extracted from some extrapolation of a non-corrected MC $S^{000}(q)$ at zero $q$. So, $\chi$ is rather derived either from the hypervirial function accumulated during the simulation ${ }^{25}$ (with a proper account of the truncated LJ force discontinuity at the cut-off $r_{c}$ ) or from discretized differentiation of the virial pressures measured at neighboring densities. We have verified that both values agree within statistical uncertainty and are consistent with the measured $p d f$ asymptote when a clear long-range plateau is available. It is also verified a posteriori that the $\chi$ value given by the exact integral equation after the inverse bridge problem resolution matches the original MC prediction. With this procedure, the remaining finite-size explicit and implicit corrections are of short-range nature. Without anticipating the results of the future paper, it is sufficient to note that the order of magnitude of these corrections is below 0.015 for $N=100$ and below 0.001 for $N=800$ for the present dipolar fluids. This last error is low enough to guarantee precise bridge functions.

As usual, the standard construction of the $p d f$ from the pair configurations gives nothing except $g(r)=0$ at short distance where the soft core repulsion prevents overlap and no information about the bridge function can be gained at $r \leq 0.85 \sigma$. Although the value of $b(r)$ in this distance regime is irrelevant for the $p d f$ and for the thermodynamics, it is still interesting to know it for different reasons, in particular here to appreciate the quality and consistency of the inverse problem resolution. The MC construction of the cavity function $y$ (rather than $g$ ) inside the core is based on the Henderson method ${ }^{24}$, described now.

\section{Cavity function inside the core}

The method is based on the Widom insertion technics ${ }^{32}$ that we briefly recall first. The excess chemical potential $\mu_{e x c}$ can be calculated by randomly inserting a test particle (labeled 0 ) inside the box, calculating the energy $v_{\text {test }}$ of that particle with all fluid particles (and, within 
the Ewald summation, with all images including its own ones and with the external medium) and statistically average the Boltzmann factor:

$$
\beta \mu_{\text {exc }}=-\ln \left\langle\exp \left(-\beta v_{\text {test }}\right)\right\rangle
$$

In fact, Widom has shown that this expression remains valid even in an inhomogeneous fluid 33: in that case, the test particle is inserted at a given position (+orientation for us) (0) and the average gives the excess chemical potential at that $(0), \beta \mu_{e x c}(0)=-\ln \left\langle\exp \left(-\beta v_{\text {test }}(0)\right)\right\rangle$. Moreover, the total chemical potential $\mu=\mu_{i d}(0)+\mu_{e x c}(0)=k T \ln (\rho(0))+\mu_{e x c}(0)$ must be uniform at equilibrium. If one formally considers that the source of the heterogeneity inside our simulation box is one of the fluid particles, say $i$, inserting the test particle at a given distance $r \equiv r_{0 i}$ and relative orientation $\Omega \equiv\left(\Omega_{0}, \Omega_{i}, r_{0 \mathrm{i}}\right)$ from $i$ gives:

$$
\beta \mu=\ln (\rho g(r, \Omega))-\ln \left\langle\exp \left(-\beta v_{\text {test }}(r, \Omega)\right)\right\rangle
$$

or, finally

$$
g(r, \Omega)=\frac{\left\langle\exp \left(-\beta v_{\text {test }}(r, \Omega)\right)\right\rangle}{\left\langle\exp \left(-\beta v_{\text {test }}\right)\right\rangle}
$$

If one is interested in the cavity function rather than the $p d f$ (that is obviously the case inside the core), it is sufficient to not count the energy of the test particle with the source $i$ (and with its images) in the energy $v_{\text {test }}$, so defining $v_{\text {test }}{ }^{*}$ :

$$
y(r, \Omega)=\frac{\left\langle\exp \left(-\beta v_{\text {test }}{ }^{*}(r, \Omega)\right)\right\rangle}{\left\langle\exp \left(-\beta v_{\text {test }}\right)\right\rangle}
$$

That is the basis of the Henderson method. In practice, one cannot avoid to first calculate the mean excess chemical potential (denominator in (16), (17)) by inserting the test particle at random positions/orientations inside the box. As usual, the statistics may be slow in dense fluids. For the construction of the numerators, different strategies have been investigated which treat the angular dependence in different manners:

i) We first accumulate $y(r, \Omega)$ for a limited number of $r$ distances (say, from 0 to $1.5 \sigma$ with $0.1 \sigma$ steps) and Euler angle triplets by inserting the test particle at the corresponding positions/orientations relative to any of the fluid particles. The price to pay is the manipulation of a big four dimension array! The reward is that the projections $\ln y^{m n l}(r)$ can be directly derived from the angular averaged of the $\ln y(r, \Omega)$ data, just in parallel to the $y^{m n l}(r)$. The Euler angles are chosen to allow for Gauss quadrature. Even if 6 values per angle seem to be sufficient, that technique corresponds to a tremendous work and the statistics is somewhat poor.

ii) In a second strategy, we do not try to construct explicitly the angle-dependence of $y$ but we rather accumulate directly, on the fly, the projections $y^{m n l}$, as it was done for $g^{m n l}$ from the pair configurations of the fluid (the $\ln y^{m n l}$ calculation being postponed in the inverse problem 
resolution). For a limited number of given distances $r$, we evaluate the different projections of the average $\left\langle\exp \left(-\beta v_{\text {test }}{ }^{*}(r, \Omega)\right)\right\rangle$ by inserting the test particle at the distance $r$ of any fluid particle $i$ and at random orientations:

$$
\left\langle\exp \left(-\beta v_{\text {test }}{ }^{*}(r, \Omega)\right)\right\rangle^{m n l}=\frac{2 l+1}{N_{\text {insertion }}} \sum_{\text {insertion }} \exp \left(-\beta v_{\text {test }}{ }^{* i}\left(r, \Omega_{0}, \Omega_{i}, \hat{r}_{0 i}\right)\right) \Phi^{m n l^{*}}\left(\Omega_{0}, \Omega_{i}, \hat{r}_{0 i}\right)
$$

$v_{\text {test }}{ }^{* i}$ must be again understood as the energy of the test particle minus the interaction with $i$.

iii) In fact, the procedure ii) can be performed directly during the standard Widom construction of the excess chemical potential without extra work: instead of fixing a priori some distances $r$, we rather use as distance information the observed distances to all fluid particles once the random insertion (random both in position and orientation) has been chosen. As before, a histogram of $r$ is constructed from the $r_{0 i}$ :

$$
\left\langle\exp \left(-\beta v_{\text {test }}{ }^{*}\right)\right\rangle^{m n l}(\operatorname{bin} k)=\frac{2 l+1}{N_{\text {insertion }} N V_{k} / L^{3}} \sum_{\text {insertion }} \sum_{\substack{i \\ r_{0 i} \in I_{k}}} \exp \left(-\beta v_{\text {test }}{ }^{* i}\left(r_{0 i}, \Omega_{0}, \Omega_{i}, \hat{r}_{0 i}\right)\right) \Phi^{m n l^{*}}\left(\Omega_{0}, \Omega_{i}, \hat{r}_{0 i}\right)
$$

A similar expression, without star label for $v_{t e s t}$, holds for the numerator of the $p d f$, (16). There is no extra cost to calculate the different $v_{\text {test }}{ }^{{ }^{i}}$. In fact, in practice, for the present dense dipolar fluids, a majority of the random insertions lead to at least two simultaneous "overlaps" and do not contribute to the denominator nor to the numerators. When the insertion leads to a single overlap, say with $i$, no information is gained for the chemical potential nor for $g$ but the $0 i$ configuration alone brings information for $y$. Lastly, for the very rare insertions without any overlap, information is available for the chemical potential as well as for $g$ and $y$ from all $0 i$ pairs.

Whatever the choice i) or iii) for the strategy, it is important to note that the "Widom" $g^{m n l}$, $y^{m n l}$ and $\ln y^{m n l}$ data will be always more noisy than the corresponding data extracted from the pair configurations, especially at high dipolar coupling and for the higher order projections. While one particle configuration gives $N(N-1) / 2$ meaningful data, one insertion gives only $N$ random data, most of them being irrelevant.

\section{Integral equation inversion}

\section{Standard integral equation resolution}

We first briefly recall the powerful technique developed for the direct numerical resolution of the $\mathrm{OZ}$ and integral equations (say HNC with or without a priori bridge prescription) in the case of non-spherically symmetric potentials ${ }^{\mathbf{3 4}} \mathbf{6}$. 
a) An iterative cycle starts from estimates of the projections $\gamma_{\alpha}\left(r_{k}\right)$ on the finite basis, $\alpha=1,2 . . \alpha_{\max }$, and discrete $r$ values, $r_{k}=k \Delta r, k=0,1, . . k_{\max }-1$ ( $k_{\max }=2048$ and $\Delta r=\sigma / 80$ here). The prescribed bridge projections, if any, are added to give the $\ln y_{\alpha}\left(r_{k}\right)$. The higher order projections of lny, $\alpha>\alpha_{\max }$, are assumed to be negligible and are ignored.

b) For each distance $r_{k}$, the $\alpha_{\max }$ first projections of the $p d f$ are derived from the pair potential and the closure (1). Calculating the projections of the exponential from those of the exponent is the most time-consuming part of the resolution. Different approximated short-cut procedures have been proposed in the literature which try to avoid angle manipulation ${ }^{6}$ but none reach the precision of the exact bare definition (the symbol $r$ is dropped for clarity):

$$
g_{\alpha}=\left\langle\exp \left[-\beta v(\Omega)+\sum_{\alpha^{\prime}=1}^{\alpha_{\max }} \ln y_{\alpha^{\prime}} \Phi_{\alpha^{\prime}}(\Omega)\right] \Phi_{\alpha}^{*}(\Omega)\right\rangle
$$

The $\ln y(\Omega)$ function is reconstructed on an array of Euler angles (molecular frame) from its projections, the potential $\beta v(\Omega)$ is subtracted, the simple exponential function is applied at each angle triplet and the result is projected back onto the desired spherical harmonics by angle averaging. We benefit from the very powerful Lado's technics which makes use of Gauss quadrature ${ }^{35}$. In practice, 20 values for each Euler angle is sufficient to guarantee a $10^{-5}$ precision in the $g_{\alpha}$. At sufficiently long distance where the exponent is small (below $10^{-5}$ ), the exponential is linearized.

c) The Hankel transform $\hat{c}^{m n l}(q)$ (of order $\left.l\right)^{\mathbf{3 4} \mathbf{6}}$, normalized by the density $\rho$, of the projection $c^{m n l}(r)=h^{m n l}(r)-\gamma^{m n l}(r)$ is calculated from spline cubic interpolation and Fast Fourier Transform (FFT) ${ }^{36}$ at the discrete $q$ values $q_{k}=k \Delta q k=0,1, . . k_{\max }-1 \quad\left(\Delta q=2 \pi / k_{\max } \Delta r\right)$. The long range $1 / r^{3}$ asymptote of $c^{112}(r) \approx-\beta v^{112}(r)$ is carefully monitored by first subtracting a reference function of identical asymptote and known Hankel transform. A signature of the dipole-dipole interaction is $\hat{c}^{112}(0) \neq 0$.

d) The molecular $\mathrm{OZ}$ equation is solved for the $\hat{\gamma}^{m n l}(q)$. The algebraic OZ equations are better expressed in the molecular frame ( $\hat{q}$ taken as the reference axis) by using the so-called $\chi$-transforms of Blum $\hat{c}_{\chi}^{m n}, \hat{\gamma}_{\chi}^{m n}$ 19. For each $\chi$ value, the matrices of the $m, n$ projections are related through ${ }^{6}$ :

$$
\begin{aligned}
& S_{\chi}(q)=1+(-1)^{\chi} H_{\chi}(q)=\left(1-(-1)^{\chi} C_{\chi}(q)\right)^{-1} \\
& \Gamma_{\chi}(q)=(-1)^{\chi} C_{\chi}^{2}(q)\left(1-(-1)^{\chi} C_{\chi}(q)\right)^{-1}
\end{aligned}
$$

e) Finally, new $\gamma_{\alpha}\left(r_{k}\right)$ values are recovered by inverse Hankel transforms of the $\hat{\gamma}_{\alpha}\left(q_{k}\right)$.

In general, input and output functions do not match and the cycle a)-e) is repeated with new, improved input. For that purpose, we have extended to anisotropic systems the full NewtonRaphson (NR) technics ${ }^{37}$ which has been proven to be very powerful in the case of spherical 
fluids or mixtures for 25 years ${ }^{38}$. The procedure which does not require any angle transformation is detailed in Appendix A. The efficiency is conserved for the present dipolar fluids and the convergence is reached quadratically as expected, in 5-10 cycles. In practice, the calculation is stopped when the difference between input and output functions vanishes below $10^{-5}$.

It is important to remember in the following that the expansion in rotational invariants is known to be less convergent for the $p d f$ or the cavity function $y$ than for the excess potential of mean force lny, mainly because of the exponentiation which emphasizes the anisotropy. So, even if the expansion of lny can be safely cut at $\alpha=\alpha_{\max }$, that does not imply that the projections $g_{\alpha}$ (or Widom $y_{\alpha}$ ), $\alpha>\alpha_{\max }$ are negligible and, indeed they are not, especially near the first peak, $r \approx \sigma$ for $g$ and at short separations, $r<\sigma$ for $y$. Ignoring them in the previous cycle just means that the higher order projections $c_{\alpha}$ would have a negligible effect on the first projections $\gamma_{\alpha}$ when injected into the molecular OZ equation (step d). This is safely true because the function $\gamma$ is a convolution product and the higher order projections $\hat{c}_{\alpha>\alpha_{\max }}(q)$ would interfere essentially in quadratic manner.

\section{Bridge function extraction}

We now impose the MC data $g_{\alpha}(r)$ (and/or Widom $y_{\alpha}(r)$ ) provided by the simulations. They are known on a limited distance $r$ regime, $r<r_{\max }$,MC, which is narrower than that required to solve the cycle, $r_{\max , \mathrm{MC}}<r_{\max }=\left(k_{\max }-1\right) \Delta r$, and on a reduced basis $\alpha \leq \alpha_{\max , \mathrm{MC}}$. Let us first describe the procedure when the number of known MC projections is identical to the number of projections required for $\ln y, \alpha_{\max , \mathrm{MC}}=\alpha_{\max }$. If the $\mathrm{MC}$ grid differs from that of the integral equation resolution, the MC data are first interpolated (spline cubic) to give the $g_{a}\left(r_{k}\right)$

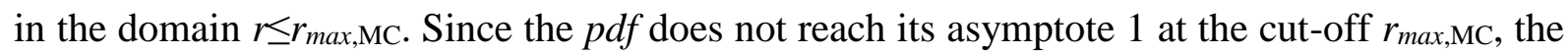
MC data must be completed at larger distances $r_{\max , \mathrm{MC}}<r \leq r_{\max }$ with some approximation. Following Verlet ${ }^{\mathbf{1 3}}$, we must choose among the bare integral equations. Two different closures have been investigated in that large separation domain, the HNC $(b=0)$ and the popular MSA $(c=-\beta v)$ chosen in numerous works ${ }^{21} 23$ within this context. Nothing can predict which choice is the best a priori. Fortunately, provided that $r_{\max , M C}$ is large enough $(5 \sigma$ is sufficient here), both approximations lead to almost identical final $b$ (mainly because $h^{2}<<b$ at large distances) and the precise choice is not critical. It is only with reduced values of $r_{\max , M C}$ that differences will emerge, see Section IV. With the HNC choice, the MC/HNC closure, step b) in the cycle, becomes:

$$
\begin{aligned}
g_{\alpha}(r) & =g_{\alpha}(r)(M C) & & ; r \leq r_{\max , M C} \\
& =\left\langle\exp \left[-\beta v(r, \Omega)+\sum_{\alpha^{\prime}} \gamma_{\alpha^{\prime}}(r) \Phi_{\alpha^{\prime}}(\Omega)\right] \Phi_{\alpha}^{*}(\Omega)\right\rangle & & ; r>r_{\max , M C}
\end{aligned}
$$

Similarly, MC/MSA reads: 


$$
\begin{array}{lll}
g_{\alpha}(r) & = & g_{\alpha}(r)(M C) \\
c_{\alpha}(r) & = & -\beta v_{\alpha}(r)
\end{array}
$$

Each approximation can be justified only a posteriori by verifying that, indeed, the bridge function $b$ or the excess direct function $c+\beta v$ is negligible at $r>r_{\max , \mathrm{MC}}$ or, equivalently, that the final $\ln y_{\alpha}(r)$ functions do not exhibit any discontinuity at $r_{\max , \mathrm{MC}}$. The rest of the cycle is unchanged and convergence is obtained as before, with straightforward modification of the NR procedure.

The final, most crucial step in the bridge extraction is to deduce at each distance $r_{k} \leq r_{\max \text {,MC }}$ the $\alpha_{\max }$ projections $\ln y_{\alpha}$ (the higher order ones being zero) from the imposed $\alpha_{\max }$ first projections $g_{\alpha}$. A naïve procedure, inverse of (20), would be:

$$
\ln y_{\alpha}=\left\langle\ln \left[\sum_{\alpha^{\prime}=1}^{\alpha_{\max }} g_{\alpha^{\prime}} \Phi_{\alpha^{\prime}}(\Omega)\right] \Phi_{\alpha}{ }^{*}(\Omega)\right\rangle+\beta v_{\alpha}
$$

with a similar expression without the final $\beta v_{\alpha}$ term when $g$ is replaced by $y$. This procedure is approximated because it implicitly ignores the non-zero, hidden, higher order projections of $g$ or $y$, and it fails, as expected, near the first peak or inside the core (some reconstructed $g(\Omega)$ take negative values in practice). So, we rather solve equation (20) in the direct way by an iterative procedure (see also ${ }^{22}$ ). We start with an estimate of the $\ln y_{\alpha}$, calculate the $g_{\alpha}$ (or $y_{\alpha}$ ) from (20), compare to the imposed MC ones and start again with improved guesses proposed by a very powerful NR technics, similar to the previous one, described in the Appendix B. Full convergence (calculated and imposed $g_{\alpha}$ match down to the machine precision!) is obtained in less than 10 cycles. At the end, the bridge projections are given by difference, $b_{\alpha}=\ln y_{\alpha}-\gamma_{\alpha}$.

The situation is more complex when the basis of $g$ provided by the simulation is more restricted than that required to describe $\ln y, \alpha_{\max , \mathrm{MC}}<\alpha_{\max }$. Without any a priori justification, we impose the HNC approximation for the missing projections (this time, MSA should be avoided because, again, the expansion in projections converges more slowly for $g$ or $c$ than for $\ln y$ or $b$ ) and the closure becomes:

$$
\begin{array}{ll}
g_{\alpha}(r)=g_{\alpha}(r)(M C) & ; \alpha \leq \alpha_{\text {max }, M C} \text { and } r \leq r_{\text {max }, M C} \\
\ln y_{\alpha}(r)=\gamma_{\alpha}(r) & ; \alpha>\alpha_{\text {max }, M C} \text { and/or } r>r_{\text {max }, M C}
\end{array}
$$

At $r>r_{\max , \mathrm{MC}}$, the situation is unchanged and the coefficients $g_{\alpha}$ are derived from the second line of (22) as before. On the other hand, at $r \leq r_{\max , \mathrm{MC}}$, the resolution requires special treatment because some projections are imposed in the exponent, other in the exponential. A robust procedure, described in Appendix $\mathrm{C}$, makes use of a new iterative cycle and generalizes the previous analysis.

In all cases, the integral equation and bridge extraction is solved in a few tens of seconds on a desk computer. 


\section{Results}

The first seven MC projections $g^{m n l}(r)$ calculated with $N=800$ are presented in figure 1 for the three dipolar couplings. The corresponding thermodynamics is given in table 1 . The dielectric constant of the external medium $\varepsilon^{\prime}$ has been chosen close to that of the dipolar fluid. The variance in the equilibrium quantities has been derived from standard block average analysis ${ }^{39}$. The less precise averages are the excess chemical potential due to the limitation of the Widom insertion technique in dense fluids and the dielectric constant $\varepsilon$ due to long-lived fluctuations in the total dipole moment $\boldsymbol{M}$ of the cell ${ }^{\mathbf{4 0}}$. Both effects are emphasized at high dipolar coupling. Note that the average $\langle\boldsymbol{M}\rangle^{2}$ which vanishes exactly in theory is measured in practice as a negligible fraction of $\left\langle\boldsymbol{M}^{2}\right\rangle$ : Typical values recorded for the ratio $\langle\boldsymbol{M}\rangle^{2} \mid\left\langle\boldsymbol{M}^{2}\right\rangle$ remain below $10^{-4}$, in agreement with the variance expected as the inverse of the number of independent configurations. The error bars in the main projections $g^{000}, g^{110}, g^{112}$, estimated at different key $r$ values using the same analysis, stay below $3.10^{-4}$ at the first peak $(r \approx \sigma)$ and below $5.10^{-5}$ at $r=L / 2=5 \sigma$, with the notable exception of $g^{110}(L / 2)$ for $\mu^{* 2}=3$ where the error bar reaches $10^{-4}$, even for such unusually long simulations. Incidentally, the insert in figure 1 which focuses on the region $r$ close to $L / 2$ may be misleading: the visual noise apparent in the curves is due to the finite width of the histogram, $\delta r=\sigma / 80$. The error bar in $g^{110}$ may be (much) higher than this noise due to long-range and long-lived collective fluctuations in the tail which are directly associated with the fluctuations in the g-factor and the dielectric constant. The different curves in figure 1 illustrate how the local arrangement inside the fluid is modified at increasing dipole-dipole coupling: the first peak in $g^{000}$ slightly shifts to lower $r$ from the pure LJ case; the negative $g^{110}$ is amplified at all distances which, according to (11), leads to a higher dielectric constant $\varepsilon$ (from 16 to 80 ); simultaneously, the projection $g^{112}$ exhibits a higher long-range $1 / r^{3}$ tail, in agreement with the theoretical prediction:

$$
g^{112}(r) \underset{r \rightarrow \infty}{\longrightarrow} \frac{\sqrt{10 / 3}(\varepsilon-1)^{2}}{4 \pi \rho y \varepsilon} \frac{1}{r^{3}}
$$

Note the weakly damped oscillations that superpose on this tail. As a consequence, internal energy, pressure, inverse compressibility and chemical potential decrease with increasing dipolar strength (Table 1). Figure 1 also clearly confirms that the expansion of the $p d f$ in projections converges very slowly: projections $g^{22 l}$ shown in the insert are not really smaller than the first ones and higher-order projections $\left(g^{13 l}, g^{33 l}, \ldots\right)$, not shown in the figure, still remain of the order unity at the first peak.

Figure 2 presents the first seven projections $y^{m n l}(r)$ of the cavity function for $\mu^{* 2}=3$ measured using the Henderson equation (technique i) in Section III, $y(r, \Omega)$ accumulated with $0.1 \sigma$ steps in $r$ ). Note the scale in the vertical axis: the function $y$ takes very high values near the origin which reveals the strong negative dipolar interaction of a particle with its neighbors inside the fluid. The corresponding curves for $\mu^{* 2}=0,1.5$ and 2.25 would be at $r \approx 0$ in the 
range $2.10^{3}, 5.10^{3}$ and $4.10^{4}$, respectively. At the origin, the projections with $l>0$ vanish while the projections with $l=0$ must present a zero slope due to the absence of hard core.

Then, the MC/HNC mixed integral equation has been solved as explained in Section III and Appendix A with $n_{\max }=2$ (7 projections) for $\mu^{* 2}=1.5$ and 2.25 or $n_{\max }=3$ (13 projections) for $\mu^{* 2}=3$. These choices guarantee that the $\gamma, \ln y$ and $b$ functions are correctly described by the finite basis and that their higher order projections can be safely ignored. In each case, we impose for the moment the same number of MC $g^{m n l}$ projections than that required for $\ln y, \alpha_{\max , \mathrm{MC}}=\alpha_{\max }$. The $\mathrm{MC}$ data are completed with the $\mathrm{HNC}$ closure beyond $r_{\max , M C}=L / 2=5 \sigma$. It should be noted that, with this $r$ cut-off, replacing MC/HNC by MC/MSA leads to virtually indistinguishable results. As a first guess for the input $\gamma$ function in the iterative resolution, the $\mathrm{HNC}$ solution is sufficient at low dipole but is too far from convergence and leads to prompt divergence at high dipole. In that last case, it is necessary to impose the MC data in the mixed closure in a softer manner by, for instance, increasing the $r_{\text {max }, M C}$ cut-off step by step from $1 \sigma$ to $5 \sigma$. As the thermodynamics is concerned, Table 1 already confirms that $\mathrm{MC} / \mathrm{HNC}$ perfectly reproduces the original $\mathrm{MC}$ data. Note the importance of properly correcting the $\mathrm{MC} p d f$ by the factor $1+\chi / N$ before use (see discussion following (13)): even if this factor is very close to $1\left(\chi / N \approx 10^{-4}\right)$, ignoring it leads to a clear discontinuity (in the $10^{-3}$ range) of $g^{000}(r)$ at $r=r_{\max , M C}$ and oscillations out of phase beyond that cut-off in the $r$-space and to spurious oscillations in the structure factor $\mathrm{S}^{000}(q)$ at $q \leq 2 \sigma^{-1}$ and incorrect compressibility $S^{000}(0)$ in the $q$-space $(\chi=0.058,0.066,0.078$ instead of the correct values $0.078,0.091,0.110$ of Table 1 , respectively). The projections $c^{m n l}(r)$ of the direct correlation function are presented in Figure 3. They are the key ingredient in any density functional theory of the solvation properties of molecular/macromolecular solutes. As for the $p d f$, the expansion converges slowly. The $c^{112}$ projection exhibits the exact long-range tail $-\beta v^{112}(r) \approx \mu^{* 2} / r^{3}$. The inserts in figure 3 enable to appreciate how quickly the function $c+\beta v$ vanishes at long distance and, thus, to what extent the MSA closure should be valid in completing MC data, see below.

The final step is to extract the $\ln y^{m n l}$ projections from those of $g$ and $y$ as described in Appendix B. Figure 4 gathers the central results of the paper and deserves detailed discussion. First, one notes that, contrary to what happened to $g, y$ or $c$, the expansion in lny converges this time very quickly. The most important coefficients are ${ }^{000},{ }^{110},{ }^{112}$ and ${ }^{022}$. The next ones like ${ }^{221}$ or ${ }^{131}$ which are clearly resolved at least for $r>0.9$ (see insert) remain below 0.05 even for $\mu^{* 2}=3$. That illustrates the fact that working with $n_{\max }=2$ or 3 is sufficient in the MC/HNC analysis (incidentally, the bare HNC requires $n_{\max } \geq 4$ for a precise determination of the dielectric constant at $\mu^{* 2}=3$; that does not contradict the previous statement because $g^{110}$ is fixed by MC within the MC/HNC). Oppositely, working with $n_{\max }=1$ (only 3 projections) would be insufficient due to the neglect of the ${ }^{022}$ coefficient and, indeed, such incomplete analysis would lead to different first $3 c$ and $\ln y$ projections. Secondly, a careful observation of the inserts in figure 4 reveals the absence of any visual discontinuity in the $\ln y^{m n l}(r)$ functions at $r=r_{\max , M C}=5 \sigma$, even at the $10^{-3}$ level, the MC data being "perfectly" prolonged by the HNC closure at larger distances. That a posteriori justifies the use of HNC in the large $r$ domain and anticipates that the bridge function is indeed negligible at distances around and 
beyond $r_{\max , M C}$. This result is especially important when one notes that the lny projections are far from being negligible in this long range domain (in particular, $\ln y^{112}$ has a $1 / r^{3}$ tail); so any simple truncation in $h$ or $\ln y$ is hopeless and it is crucial to be able to complete the MC data with correct closure at larger distances. Third, the branches $r<r^{*} \approx 0.87$ and $r>r^{*}$ which correspond to the use of the MC $y$ and $g$ data, respectively, agree clearly each other. We have used the three separate techniques described in Section III for constructing the $y$ function and all lead to consistent lny projections. It is obvious that the $y$ data are much noisier than the $g$ data due to the standard limitation of the Widom insertion technique, especially for the highest dipole. So, the $\ln y^{m n l}$ projections coming from $y$ are less precise. The estimated uncertainty for $\mu^{* 2}=3$ is visualized by the size of the symbols in figure 4 . Within this noise, both branches connect with precision at $r^{*}$ for the first 4 projections and coincide in the overlapping region $r=0.87-1.5$. In parallel, the higher-order projections $\ln y^{22 l}$ at $r<r^{*}$ cannot be distinguished from the noise. The overall agreement between $y$ and $g$ sets of data validates the analysis. As counterexample, working with $n_{\max }=1$ would lead to clear discontinuities of the order of 0.5 in the first $3 \ln y$ projections at $r^{*}$ !

Lastly, subtracting $\gamma$ from lny leads to the desired projections $b^{m n l}(r)$ of the bridge function, plotted in figure 5. The expansion in $b$ converges equally well. The coefficients $b^{13 l}$ are negligible for $\mu^{* 2}=1.5$ and 2.25 and remain below 0.04 for $\mu^{* 2}=3$. At large distances, the $b$ projections vanish much faster than the lny ones (compare the inserts of figures 4 and 5). They remain below $4.10^{-4}$ at $r>4 \sigma$. That very small value, consistent with the previously noted absence of discontinuity in the lny curves, validates the use of the HNC approximation beyond the cut-off $r_{\max , M C}=5 \sigma$ and confirms that a truncation in $b$ is better than in $h$ or in $\ln y$. At shorter distances, the $y$-originated data inside the core are affected by the previously discussed statistical noise but remain consistent with the $g$-originated data at $r^{*}$. Note that the $b^{22 l}$ projections for $\mu^{* 2}=3$ are clearly visible near the origin, contrary to what happened to $\ln y$; that means that they are nearly opposite to the corresponding $\gamma^{22 l}$ projections.

It is difficult if not meaningless to derive some hypothetic universality law from the whole $b$ curves of figure 5. Even if the general shape of $b^{000}$ looks like that of the bridge function for spheres (see the pure LJ curve), the complex next-order projections are specific to dipolar systems and reveal the strong anisotropy of the bridge function. As an illustration, $b(r, \Omega)$ can be constructed from these projections and (3) for some characteristic relative orientations $\Omega$ and plotted as a function of $r$, see figure 6 for the highest dipole. The different curves present very different shapes in the relevant domain $r \approx 1-3$. We note in particular the clear peak at $r \approx 2 \sigma$ for the aligned/oriented configuration of the two dipoles which results from the corresponding peaks in the different projections in figure 5; that reveals the difficulty of the bare $\mathrm{HNC}$ closure (which entirely neglects the bridge) to deal with favorable configurations of aligned triplets of dipoles near contact.

In order to test and compare the relative merits of the MSA and HNC closures in completing the MC data at large distances, the $c+\beta v$ and $b$ projections have been plotted for $\mu^{* 2}=3$ using the same scale in the inserts of figure 3 and 5, respectively. First, one notes again that both sets of projections are equally negligible (less than a few $10^{-4}$ ) at $r>4 \sigma$, thus explaining why 
$\mathrm{MC} / \mathrm{HNC}$ and MC/MSA treatments are identically "exact" with the choice $r_{\max , M C}=5 \sigma$. Moreover, the function $c+\beta v$ clearly vanishes faster than $b$ in the intermediate region $r=2-5$ for this high dipolar coupling. To the author's knowledge, this important observation could not be anticipated from theoretical arguments. On one side, it is well known that the bare MSA closure, of linearized nature, gives very bad results at high $\mu^{* 2}$ while the bare HNC closure behaves quite well; so, the opposite trend $c+\beta v>>b$ applies near the first peak. On the other side, in absence of known theoretical asymptotic law, one could just say that an expansion of (1) gives $c+\beta v \approx b+1 / 2 h^{2} \ldots$ for the tail. At large distances, if $h^{2}<<b$, then $c+\beta v$ and $b$ coincide. At intermediate distances, the $1 / r^{3} h^{112}$ tail (26) dominates in $h$ and contributes to $1 / r^{6}$ non negligible terms in some projections of $h^{2}\left({ }^{000},{ }^{022} \ldots\right)$, especially at high dielectric constant. The fact that $c+\beta v<<b$ in practice just means that these terms are recovered in $b$ with the factor $-1 / 2$. In order to illustrate the present observation and to bring MSA and HNC closures against more severe tests, we have performed again the MC/HNC and MC/MSA treatments for $\mu^{* 2}=3$ but with limited sets of MC data characterized by lower values of $r_{\max , M C}<5 \sigma$. Figure 7 corresponds to the choice $r_{\max , M C}=3 \sigma$. In the domain $r=3-5 \sigma$, both mixed equations reproduce with very good accuracy the original $\ln y^{m n l} \mathrm{MC}$ data of figure 3, with nevertheless clear advantage for MC/MSA. This is not unexpected since we knew that $c+\beta v<0.001$ and $b<0.003$ at $r>3 \sigma$. Table 2 gives the same kind of almost perfect agreement for the thermodynamics (the attentive reader may have noticed that the dielectric constant decreases from 81 to 80 when going from $r_{\max , M C}=5 \sigma$ to $3 \sigma$ and becomes closer to the original $\mathrm{MC}$ value; that small but relevant effect is not fortuitous and illustrates the fact that the $\mathrm{MC}$ $p d f$ is affected by finite-size implicit corrections at large distances, close to $L / 2^{28}$ ). Figure 8 goes further and investigates the more severe choice $r_{\max , M C}=1.8 \sigma$, both in $h^{m n l}$ and in $b^{m n l}$. This time, the $\mathrm{MC} / \mathrm{HNC}$ treatment ignores by construction the previously discussed peak of the bridge projections localized around $2 \sigma$, of height in the range 0.05 (see insert of figure 5). As a consequence, the original $\mathrm{MC} p d f$ is not well reproduced at $r>1.8 \sigma$, the dielectric constant is grossly overestimated (Table 2) and the bridge function becomes qualitatively incorrect, even below the cut-off, $r<1.8 \sigma$ (insert of figure 8 ). On the other hand, since the $c+\beta v$ projections were bounded in the 0.01 range at $r \approx 2 \sigma$ (see insert of figure 3 ), the MC/MSA treatment with this reduced cut-off remains very powerful in reproducing the $p d f$, the thermodynamics (Table 2) and the whole bridge function, including its details at $r \approx 2 \sigma$. To conclude this analysis, despite existing long-range correlations in the fluid, it is sufficient to impose only "short-range" MC data and to complete with HNC or, better, with MSA. That also opens the possibility to work with much smaller simulation cells $(L / 2=2.5 \sigma$ corresponds to $N=100$ ), provided that the MC are beforehand correctly cleaned from non-negligible finitesize corrections ${ }^{28}$.

To what extent is it necessary to impose all available MC projections? Is it sufficient to accumulate and impose a few first-order MC $g^{\text {mnl }}$ only and to complete with the HNC closure for the higher-order ones (remember, MSA is not valid in this context)? To answer these questions, we have solved the new MC/HNC (25) for $\mu^{* 2}=3$ with a number of fixed MC projections, $\alpha_{\max , \mathrm{MC}}$, smaller than that required to describe $\ln y, \alpha_{\max }=13$. The numerical resolution follows the procedure described in Appendix C. Figures 9 (for the $p d f$ ) and 10 (for 
the bridge) and Table 3 summarize the results for $\alpha_{\max , \mathrm{MC}}=3$ (MC $g^{000}, g^{110}, g^{112}$ fixed), $\alpha_{\max , \mathrm{MC}}=1$ (MC $g^{000}$ fixed only) and $\alpha_{\max , \mathrm{MC}}=0$ (no MC projection fixed at all, bare HNC). The RHNC approximation which identifies the bridge function with that of the naked pure LJ fluid is added for comparison. First, it is clear that HNC and RHNC fail to describe properly the details of all $p d f$ projections and the thermodynamics. Secondly, $\mathrm{MC} / \mathrm{HNC}\left(\alpha_{\max , \mathrm{MC}}=1\right)$ which, by definition, reproduces exactly the center of mass $g^{000}$ of the dipolar fluid, brings no improvement for the higher-order $p d f$ projections. That again illustrates the importance of the complete bridge function and its non-universal anisotropic character. Third, $\operatorname{MC} / \mathrm{HNC}\left(\alpha_{\max , \mathrm{MC}}=3\right)$ which accounts for this anisotropy through non zero $b^{110}$ and $b^{112}$ terms, matches much better the exact $\mathrm{MC}$ or $\mathrm{MC} / \mathrm{HNC}\left(\alpha_{\max , \mathrm{MC}}=13\right)$ data. For the thermodynamics, that is not unexpected since energy, pressure, compressibility and dielectric constant depend on the first three $p d f$ projections only. Concerning the higher-order $g^{m n l}$ terms, figure 9 reveals good success for ${ }^{022},{ }^{220}$ and ${ }^{222}$ and quantitative disagreement for ${ }^{224}$. Since all projections are mixed in the exponential (1) in a complex manner, imposing exact first three $g^{m n l}$ does not guarantee exact first three $b^{m n l}$ and, indeed, figure 10 illustrates how $b^{000}, b^{110}$ and $b^{112}$ deviate from the exact reference functions in the relevant region, $r=1-2 \sigma$. These small but clear effects arise from the neglect of the next-order $b^{m n l}$ projections (resolved in the inserts of figure 5) near the first peak and close to $r \approx 2 \sigma$.

\section{Conclusion}

With the help of powerful techniques specifically designated for the case of strongly anisotropic correlations, it has been possible to invert the $\mathrm{OZ}$ and integral equations and to extract the bridge function from exact MC $p d f$ data for the dipolar Stockmayer fluid. The expansion in rotational invariants of the negative excess potential of mean force $\ln y(r)$ and of the bridge $b(r)$ converges quickly and a few projections (7-13) are sufficient to account for the orientation dependence, even at high dipole coupling. A characteristic peak at $r \approx 2 \sigma$ is the signature of configurations of aligned dipole triplets near contact. The presence of long-range electrostatic correlations requires completing the $\mathrm{MC}$ data with approximated closures at large distances. HNC and MSA perform very well at $r>3 \sigma$ while MSA remains astonishingly good at $r>1.8 \sigma$. That opens the possibility to work with reduced numbers of dipoles in the simulation cell. The extension of the present analysis to more realistic models of water-like systems will deal with non-linear geometries and five Euler angles or five indices $g^{m n l} \mu \nu$ descriptions. The procedure will be essentially unchanged and the increased difficulty will be of pure numerical nature.

\section{Acknowledgment}

Support from ANR under grant No.ANR-06-BLAN-0276 is gratefully acknowledged. 


\section{Appendix A. Newton-Raphson algorithm for the integral equation resolution}

The main cycle a)-e) in III for the iterative resolution of the $\mathrm{OZ}+$ closure equations starts with input $\gamma_{\alpha}{ }^{(\mathrm{in})}\left(r_{k}\right)$ and finishes with output $\gamma_{\alpha}{ }^{\text {(out) }}\left(r_{k}\right)$ values. Not too far from the convergence, we assume that the exact solution $\gamma^{(\text {in) }}+\mathrm{d} \gamma^{\text {(in) }}=\gamma^{\text {(out) }}+\mathrm{d} \gamma^{\text {(out) }}$ (array notation) is sufficiently close to $\gamma^{\text {(in) }}$ to express $\mathrm{d} \gamma^{\text {(out) }}$ linearly in function of $\mathrm{d} \gamma^{\text {(in) }}, \mathrm{d} \gamma^{\text {(out) }}=\mathbf{A d} \gamma^{\text {(in) }}$. The operator $\mathbf{A}$ is deduced from the main cycle by mathematical differentiation of each step a)-e), see below. The NR technics then consists to solve the linear system (1-A) $X=B$ where $X=\mathrm{d} \gamma^{\text {(in) }}$ and $B=\gamma^{\text {(out) }}-\gamma^{(\text {in) }}$. Since it is impossible in practice to invert the large matrix 1-A of size $\left(\alpha_{\max } k_{\max } \times \alpha_{\max } k_{\max }\right)$, the linear system is solved by iteration using the powerful conjugate gradient method proposed by Zerah in the context of integral equations ${ }^{37}$ and used with great success for 25 years in various spherical liquids and mixtures ${ }^{\mathbf{3 8}}$. Roughly speaking, each new cycle requires one calculation $\mathbf{A} X$ for some $X$ and one $\mathbf{A}^{\mathbf{T}} Y$ for some $Y$. The success of the procedure is directly linked to the convergence, as fast and complete as possible, of the linear cycle. The operator $\mathbf{A}^{\mathbf{T}}$ must be written as the exact numerical transpose of $\mathbf{A}$ (in particular, the FT with spline cubic interpolation is not self-transpose contrary to a bare FFT) and the effect of the round-off errors is minimized by working in double precision. For the present study, the linear cycle converges in practice in $\approx 50$ iterations for the HNC equation and in $\approx 200$ iterations when MC noisy data are imposed. Once the convergence is attained for $X$, a new main cycle is started with the improved input function $\gamma^{(i n)}+X$.

The OZ step d) of the operator $\mathbf{A}$ is given by the linearization of (21):

$$
d \Gamma_{\chi}=S_{\chi} d C_{\chi} S_{\chi}-d C_{\chi}
$$

The crucial step b) of the operator $\mathbf{A}$ is defined by the linearization of the exponential closure (1) which reads at each distance $r$ (implicit in the following equations):

$$
d g(\Omega)=g(\Omega) d \gamma(\Omega)
$$

The key is to derive the projections of the product $d g$ from those of each term $g, d \gamma$ without time-consuming angle-triplet manipulation (so, the closure is not linearized in its (20) form). From the known expansion of the product of two rotational invariants, $\Phi_{\alpha_{1}} \Phi_{\alpha_{2}}=\sum_{\alpha} t_{\alpha_{1} \alpha_{2} \alpha} \Phi_{\alpha}$ with well-documented coefficients $t^{\mathbf{2 0}}$, the desired relation reads:

$$
d g_{\alpha}=\sum_{\alpha_{1}, \alpha_{2}} t_{\alpha_{1} \alpha_{2} \alpha} g_{\alpha_{1}} d \gamma_{\alpha_{2}}
$$

It is important to remember again that even if the basis of $d \gamma$ is restricted to $\alpha_{2} \leq \alpha_{\text {max }}$ and the required projections of $d g$ are limited to $\alpha \leq \alpha_{\max }$, the projections of $g$ which are relevant in (29) go beyond this same basis. Ignoring this fact and restricting $\alpha_{1} \leq \alpha_{\max }$ in the products (29) leads to an operator $\mathbf{A}$ which is not exactly the derivative of the main cycle. That does not affect the convergence of the NR linearized cycle but the new input $d \gamma$ function proposed by the algorithm is not as improved as it should be and the main convergence is slower than 
expected. In order to recover the full NR efficiency and the very fast, quadratic convergence of the main cycle, it is necessary to account for all $g$ projections relevant in (29) (non-zero $t$ coefficients). If the working basis is defined by $m, n \leq n_{\max }$, that corresponds to choosing $m_{1}, n_{1} \leq 2 n_{\max }$ or $\alpha_{1} \leq \alpha_{\max }$. This necessitates calculating at each Lado's step (20) high-order projections of $g$. For the present linear dipolar molecules, the extra cost is modest: if $n_{\max }=2$, 15 extra projections are required, $\alpha_{\max }=22$. This would not be as simple for non-linear, waterlike geometry!

\section{Appendix B. Iny projections from Monte Carlo $g$ projections}

At each distance $r$, we are looking for the $\alpha_{\max }$ projections of lny (the higher order ones being zero) which lead to the first $\alpha_{\max }$ projections of $g$ imposed by the simulation, $g_{\alpha} \mathrm{MC}$. In case of $y$ (Widom) MC imposed data, it is sufficient to replace $g$ by $y$ and to discard the potential term in the closure. Equation (20) is solved iteratively with NR technics. At a given iteration, lny produces $g$, different from $g^{\mathrm{MC}}$. An increase dlny of lny would produce (linear expansion) the increase in $g$ given by an equation similar to (29):

$$
d g_{\alpha}=\sum_{\alpha_{1}, \alpha_{2}} t_{\alpha_{1} \alpha_{2} \alpha} g_{\alpha_{1}} d \ln y_{\alpha_{2}}=\sum_{\alpha_{2}=1}^{\alpha_{\max }}\left(\sum_{\alpha_{1}=1}^{\alpha_{\max }{ }^{\prime}} t_{\alpha_{1} \alpha_{2} \alpha} g_{\alpha_{1}}\right) d \ln y_{\alpha_{2}} \equiv \sum_{\alpha_{2}=1}^{\alpha_{\max }} M_{\alpha, \alpha_{2}} d \ln y_{\alpha_{2}}
$$

or, in matrix notation, $d g=(\boldsymbol{M}) \mathrm{d} \ln y$. Once again, it is crucial to account for all relevant $g$ projections, $\alpha_{1} \leq \alpha_{\max }$, in the construction of the matrix $\boldsymbol{M}$. If not, the procedure would completely fail (no NR convergence or, even divergence) near the first peak or inside the core. The NR step consists to start the next iteration with the improved solution, $\ln y+\boldsymbol{M}^{-}$ ${ }^{1}\left(g^{\mathrm{MC}}-g\right)$. The first estimate is chosen from a previous neighboring case, from the approximate equation (24) or, if not sufficient, from linear extrapolation of higher distances $r$, previously treated with more ease. Once the first estimate is close enough to the true solution, the NR quadratic convergence is very fast and calculated and imposed $g$ functions agree down to the machine double precision.

\section{Appendix C. Iny projections from limited Monte Carlo $g$ information}

This time, the simulation provides a limited number of $g_{\alpha}$ projections, $\alpha_{\max , \mathrm{MC}}<\alpha_{\max }$ and the HNC closure is assumed for the higher order projections, (25). Since the exponential operation mixes known and unknown projections at $r \leq r_{\max , \mathrm{MC}}$, the resolution cannot be as "simple" as in the previous cases and must use an extra iterative resolution. After many trials, the most stable algorithm seems to be the following.

1) An estimate is made for the projections $\gamma_{\alpha}, \alpha>\alpha_{\max , \mathrm{MC}}$ at $r \leq r_{\max , \mathrm{MC}}$ and fixed during step 2). 
2) At each distance $r \leq r_{\max , \mathrm{MC}}$, we extract the $\alpha_{\max , \mathrm{MC}}$ first projections of lny which lead to the imposed $\alpha_{\max , \mathrm{MC}}$ first projections of $g^{\mathrm{MC}}$ through (20), at fixed $\ln y_{\alpha} \equiv \gamma_{\alpha}, \alpha>\alpha_{\max , \mathrm{MC}}$ of step 1). The procedure is identical to that described in Appendix B. These projections are then fixed during step 3).

3) We solve in terms of the $\gamma_{\alpha}$ projections the integral equation problem with closure:

$$
\begin{array}{ll}
\ln y_{\alpha}(r)=(\operatorname{step} 2) & ; \alpha \leq \alpha_{\text {max }, M C} \text { and } r \leq r_{\text {max }, M C} \\
\ln y_{\alpha}(r)=\gamma_{\alpha}(r) & ; \alpha>\alpha_{\text {max }, M C} \text { and/or } r>r_{\text {max }, M C}
\end{array}
$$

This time, all projections of the potential of mean force are described simultaneously and the resolution is similar to the previous ones. At convergence, the $\alpha_{\text {max,MC }}$ first projections of $g$ are (slightly) different from the MC data because the $\gamma_{\alpha}, \alpha>\alpha_{\max , \mathrm{MC}}$ have changed between steps 1) and 3).

4) The procedure is repeated at step 1) with the new $\gamma_{\alpha}, \alpha>\alpha_{\max , \mathrm{MC}}$ of step 3).

In practice, the full convergence is obtained in 2-4 extra iterations. 
Table 1. Thermodynamics

\begin{tabular}{|c|c|c|c|c|c|c|c|c|}
\hline$\mu^{* 2}$ & & $N_{\text {cycles }}$ & $\varepsilon^{\prime}$ & $-U / N k T$ & $P / \rho k T$ & $\chi$ & $\beta \mu_{e x}$ & $\varepsilon$ \\
\hline 1.5 & HNC & & & 3.6059 & 3.638 & 0.130 & 2.152 & 15.52 \\
\hline & MC & $510^{6}$ & 16 & $3.7927 \pm 10^{-4}$ & $2.535 \pm 10^{-3}$ & 0.0785 & $0.755 \pm 0.005$ & $16.4 \pm 0.1$ \\
\hline & MC/HNC & & & 3.793 & 2.538 & 0.0790 & & 16.30 \\
\hline 2.25 & HNC & & & 5.004 & 2.883 & 0.159 & 0.325 & 33.32 \\
\hline & MC & $110^{7}$ & 37 & $5.2118 \pm 10^{-4}$ & $1.778 \pm 10^{-3}$ & 0.0925 & $-1.10 \pm 0.01$ & $36.9 \pm 0.1$ \\
\hline & MC/HNC & & & 5.2120 & 1.783 & 0.091 & & 37.2 \\
\hline 3 & HNC & & & 6.603 & 2.158 & .217 & -1.636 & 92.3 \\
\hline & MC & $110^{7}$ & 80 & $6.8045 \pm 10^{-4}$ & $1.064 \pm 10^{-3}$ & 0.110 & $-3.10 \pm 0.03$ & $80.0 \pm 0.5$ \\
\hline & MC/HNC & & & 6.805 & 1.066 & 0.111 & & 81.0 \\
\hline
\end{tabular}

Within the MC/HNC approach, the MC data $(N=800)$ are imposed at $r<r_{\max , M C}=5 \sigma$ and completed by the HNC closure beyond $r_{\max , M C} \cdot \varepsilon^{\prime}$ is the imposed dielectric constant of the external medium in the Ewald summation; the pressure $P$ is derived from the virial equation; the compressibility $\chi$ is calculated from the hypervirial equation (MC) or from $S(0)$ (HNC and $\mathrm{MC} / \mathrm{HNC}$ ). The excess chemical potential $\mu_{e x}$ is obtained from the Widom insertion technique (MC) or from integral of correlation functions (HNC). The dielectric constant of the medium $\varepsilon$ is derived from (10) and the total moment fluctuations $\left\langle M^{2}\right\rangle$ of the cell (MC) or from the Kirkwood equation $\left((10)\right.$ with $\left.\varepsilon^{\prime}=\varepsilon\right)$ with the g-factor given by the integral (11) of $g^{110}(r)$ up to infinity. 
Table 2. Thermodynamics for $\mu^{* 2}=3$ - Limited MC $r_{\max , M C}$ cut-off

\begin{tabular}{|c|c|l|c|c|c|c|c|}
\hline$r_{\max , M C}$ & & & & $-U / N k T$ & $P / \rho k T$ & $\chi$ & $\varepsilon$ \\
\hline & MC & & & $6.8045 \pm 10^{-4}$ & $1.064 \pm 10^{-3}$ & 0.110 & $80.0 \pm 0.5$ \\
\hline $5 \sigma$ & MC/HNC & & 6.805 & 1.066 & 0.111 & 81.0 \\
\hline $3 \sigma$ & MC/HNC & & 6.805 & 1.069 & 0.112 & 80.0 \\
\hline & MC/MSA & & 6.805 & 1.069 & 0.110 & 79.9 \\
\hline $1.8 \sigma$ & MC/HNC & & 6.813 & 1.062 & 0.123 & 97 \\
\hline & MC/MSA & & 6.805 & 1.069 & 0.109 & 80.1 \\
\hline
\end{tabular}

Within the MC/HNC and MC/MSA approaches solved with $\alpha_{\max , M C}=13$ projections, the MC data are imposed at $r<r_{\max , M C}$ and completed by the HNC and MSA closures beyond $r_{\max , M C}$, respectively. 
Table 3. Thermodynamics for $\mu^{* 2}=3$ - Limited number $\alpha_{\max , M C}$ of imposed MC projections in the MC/HNC model

\begin{tabular}{|c|c|c|c|c|}
\hline$\alpha_{\max , M C}$ & $-U / N k T$ & $P / \rho k T$ & $\chi$ & $\varepsilon$ \\
\hline 13 & 6.805 & 1.066 & 0.111 & 81.0 \\
\hline 3 & 6.806 & 1.068 & 0.111 & 81.0 \\
\hline 1 & 6.596 & 1.275 & 0.111 & 68 \\
\hline RHNC & 6.632 & 0.681 & 0.101 & 77 \\
\hline
\end{tabular}

The MC/HNC model is solved with $\alpha_{\max }=13$ projections. The MC data are imposed up to $r_{\max , M C}=5 \sigma$. The RHNC integral equation identifies the bridge function $b=b^{000}$ with that of the pure LJ fluid. 


\section{Figure caption}

\section{Figure 1:}

Main panel and upper right insert: MC projections $g^{m n l}(r)$ of the $p d f$ for $\mu^{* 2}=1.5$ (dotted lines), 2.25 (dashed) and 3 (solid) constructed with $N=800$ particles. The thin solid line corresponds to the naked LJ fluid, $\mu^{* 2}=0$. The bottom right insert focuses on the large $r$ region, close to $L / 2=5 \sigma$, for $\mu^{* 2}=3$.

\section{Figure 2:}

MC projections $y^{m n l}(r)$ of the cavity function derived from the Widom-Henderson insertion technique for $\mu^{* 2}=3$ (symbols). The lines are guide for the eyes.

\section{Figure 3:}

$\mathrm{MC} / \mathrm{HNC}$ projections $c^{m n l}(r)$ of the direct correlation function. Calculation with $\alpha_{\max }=\alpha_{\max , \mathrm{MC}}=7$ projections $\left(\mu^{* 2}=1.5\right.$ and 2.25$)$ or $=13$ projections $\left(\mu^{* 2}=3\right)$. MC data are imposed up to $r_{\max , M C}=5 \sigma$. Same legend as figure 1 . The inserts give the first projections of $c+\beta v$ for $\mu^{* 2}=3$.

\section{Figure 4:}

$\mathrm{MC} / \mathrm{HNC}$ projections $\ln y^{m n l}(r)$ of the negative excess potential of mean force. Same legend as figures 1 and 3. The arrow at $r^{*} \approx 0.87 \sigma$ separates the two domains $r<r^{*}$ and $r>r^{*}$ where $\ln y$ is constructed from the MC Widom $y$ and the MC $g$, respectively. For $\mu^{* 2}=3$, the $y$-originated data are marked by symbols whose size is of the order of the uncertainty. The two inserts focus on the tails for $\mu^{* 2}=3$, with the arrow representing $r_{\max , M C}=5 \sigma$.

\section{Figure 5:}

MC/HNC projections $b^{m n l}(r)$ of the bridge function. Same legend as figure 4 . The thin solid line corresponds to the naked LJ fluid, $\mu^{* 2}=0$. The two inserts focus on the case $\mu^{* 2}=3$ and use the same scale as in the corresponding inserts of figure 3.

\section{Figure 6:}

MC/HNC bridge function $b(r, \Omega)$ at $\mu^{* 2}=3$ for some characteristic orientations $\Omega$ pictured on the figure: the arrows indicate dipole orientations; the dot is for a dipole pointing out of the figure. Same legend as figure 4.

\section{Figure 7:}

$\mathrm{MC} / \mathrm{HNC}$ and MC/MSA projections $\ln y^{m n l}(r)$ at $\mu^{* 2}=3 . \quad r_{\max , M C}=5 \sigma$, reference symbols; $r_{\max , M C}=3 \sigma$ (arrow), continuous lines (MC/HNC) and dashed lines (MC/MSA), mostly hidden behind the symbols. 
Figure 8:

$\mathrm{MC} / \mathrm{HNC}$ and MC/MSA projections $h^{m n l}(r)$ and $b^{m n l}(r)$ (insert) at $\mu^{* 2}=3 . r_{\max , M C}=5 \sigma$, reference symbols; $r_{\max , M C}=1.8 \sigma$ (arrow), continuous lines $(\mathrm{MC} / \mathrm{HNC}$ ) and dashed lines (MC/MSA), mostly hidden behind the symbols.

\section{Figure 9:}

Projections $g^{m n l}(r)$ of the $p d f$ at $\mu^{* 2}=3$. MC data (symbols); bare HNC (solid line); RHNC (bridge function borrowed from the pure LJ fluid, dotted line); MC/HNC (25) resolved $\left(\alpha_{\max }=13\right)$ with a limited set of imposed MC $g^{\operatorname{mnl}}$ projections up to $r_{\max , M C}=5 \sigma . \alpha_{\max , M C}=1$ (dashed) or $\alpha_{\max , M C}=3$ (dotted-dashed).

\section{Figure 10:}

$\mathrm{MC} / \mathrm{HNC}$ projections $b^{m n l}(r)$ of the bridge function at $\mu^{* 2}=3 . \alpha_{\max }=13$. The number $\alpha_{\max , M C}$ of imposed MC $g^{m n l}$ projections up to $r_{\max , M C}=5 \sigma$ is 13 (reference symbols), 3 (dotted-dashed lines) or 1 (dashed line). The dotted line is the bridge function of the pure LJ fluid. 


\section{References}

1. J. P. Hansen and I. R. McDonald. Theory of Simple Liquids; Academic: London, 1986.

2. D. Levesque, Physica 32, 1985 (1966)

3. J. P. Hansen and I. R. McDonald, Phys. Rev. A 11, 2111 (1975)

4. J. C. Rasaiah and H. L. Friedman, J. Chem. Phys. 48, 2742 (1968)

5. L. Belloni, Chem. Phys. 99, 43 (1985)

6. P. H. Fries and G. N. Patey, J. Chem. Phys. 82, 429 (1985)

7. Y. Rosenfeld and N. W. Ashcroft, Phys. Rev. A 20, 1208 (1979)

8. P. Attard and G. N. Patey, J. Chem. Phys. 92, 4970 (1990)

9. F. J. Rogers and D. A. Young, Phys. Rev. A 30, 999 (1984)

10. G. Zerah and J. P. hansen, J. chem. Phys. 84, 2336 (1986)

11. J. L. Barrat, J. P. Hansen, and G. Pastore, Mol. Phys. 63, 747 (1988)

12. P. Attard, J. Chem. Phys. 95, 4471 (1991)

13. L. Verlet, Phys. Rev. 163, 201 (1968)

14. R. Wedberg, J. P. O'Connell, G. H. Peters, and J. Abildskov, Fluid Phase Equilib. 302, 32 (2011)

15. M. Llano-Restrepo and W. G. Chapman, J. Chem. Phys. 97, 2046 (1992)

16. M. Llano-Restrepo and W. G. Chapman, J. Chem. Phys. 100, 5139 (1994)

17. J. Kolafa, S. Labik, and A. Malijevsky, Mol. Phys. 100, 2629 (2002)

18. L. Blum and A. J. Torruella, J. Chem. Phys. 56, 303 (1972)

19. L. Blum, J. Chem. phys. 57, 1862 (1972)

20. A. Messiah. Quantum Mechanics; Wiley: New York, 1962; Vol. II.

21. D. L. Cheung, L. Anton, M. P. Allen, and A. J. Masters, Phys. Rev. E 73, 61204 (2006)

22. D. L. Cheung, L. Anton, M. P. Allen, A. J..P.J. Masters, and M. Schmidt, Phys. Rev. E 78, 
$41201(2008)$

23. R. Ramirez, M. Mareschal, and D. Borgis, Chem. Phys. 319, 261 (2005)

24. J. R. Henderson, Mol. Phys. 48, 389 (1983)

25. M. P. Allen and D. J. Tildesley. Computer Simulation of Liquids; Oxford University Press: New York, 1987.

26. M. Neumann and O. Steinhauser, Chem. Phys. Lett. 95, 417 (1983)

27. S. W. de Leeuw, J. W. Perram, and E. R. Smith, Proc. R. Soc. Lond. A 373, 57 (1980)

28. L. Belloni and J. Puibasset, to be published

29. J. M. Caillol, J. Chem. Phys. 96, 7039 (1992)

30. J. L. Lebowitz and J. K. Percus, Phys. Rev. 124, 1673 (1961)

31. L. R. Pratt and S. W. Haan, J. Chem. Phys. 74, 1864 (1981)

32. B. Widom, J. Chem. Phys. 39, 2808 (1963)

33. B. Widom, J. Stat. Phys. 19, 563 (1978)

34. F. Lado, Mol. Phys. 47, 283 (1982)

35. J. A. Anta, E. Lomba, C. Martin, M. Lombardero, and F. Lado, Mol. Phys. 84, 743 (1995)

36. N. O. Einarsson, BIT 8, 279 (1968)

37. G. Zerah, J. Comput. Phys. 61, 280 (1985)

38. L. Belloni, J. Chem. Phys. 88, 5143 (1988)

39. H. Flyvbjerg and H. G. Petersen, J. Chem. Phys. 91, 461 (1989)

40. P. G. Kusalik, Mol. Phys. 73, 1349 (1991) 
Figure 1

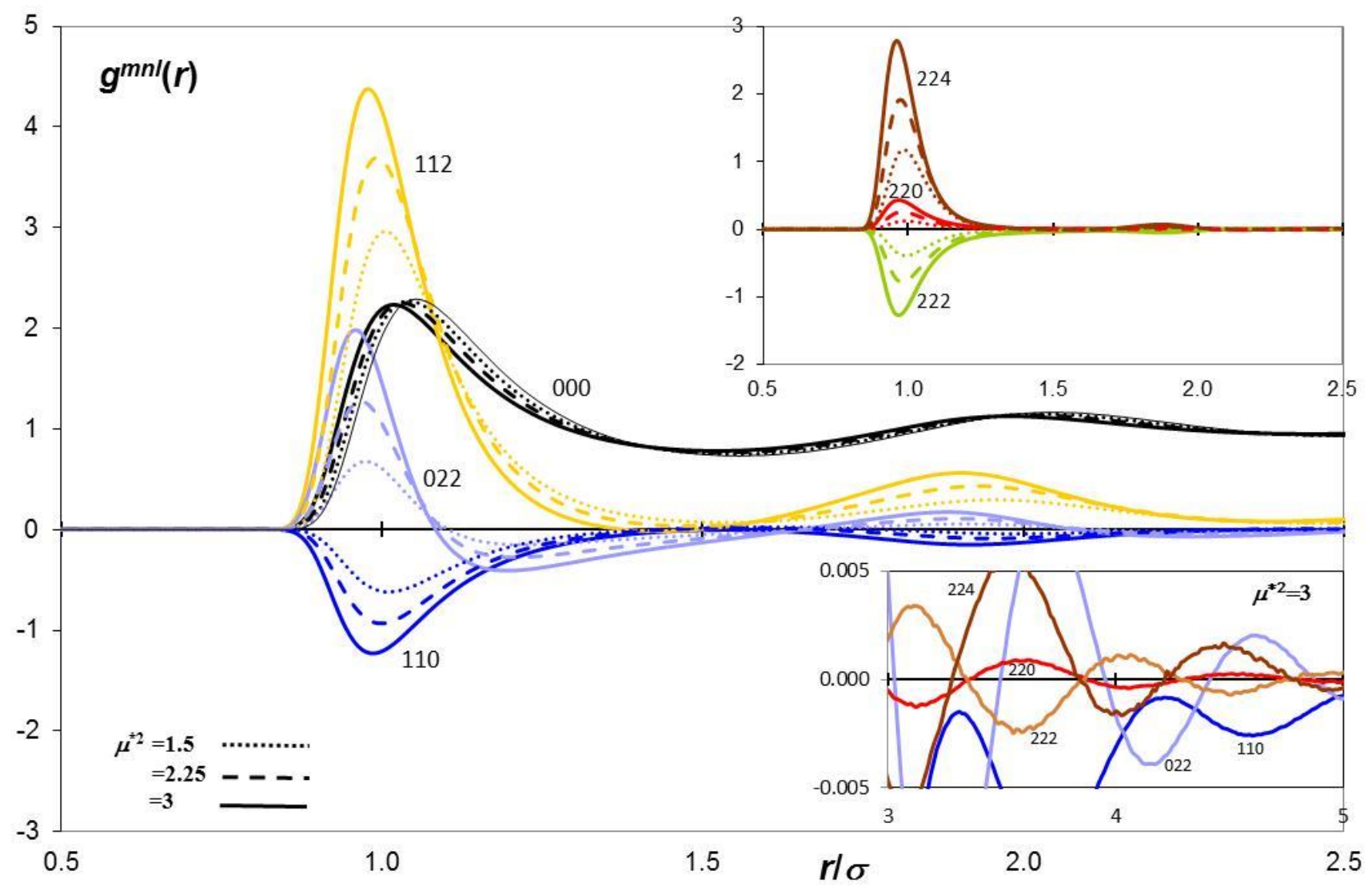


Figure 2

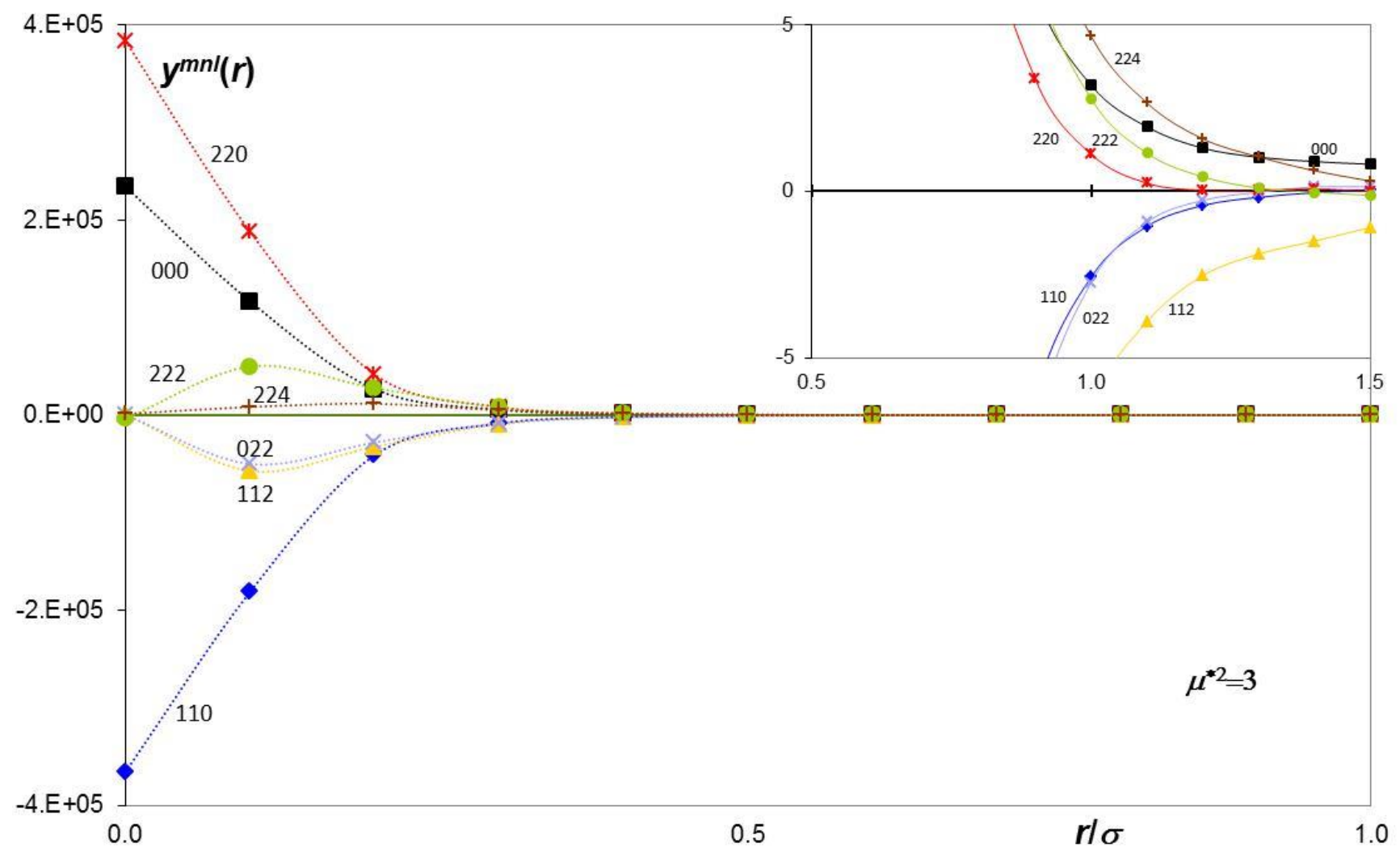


Figure 3

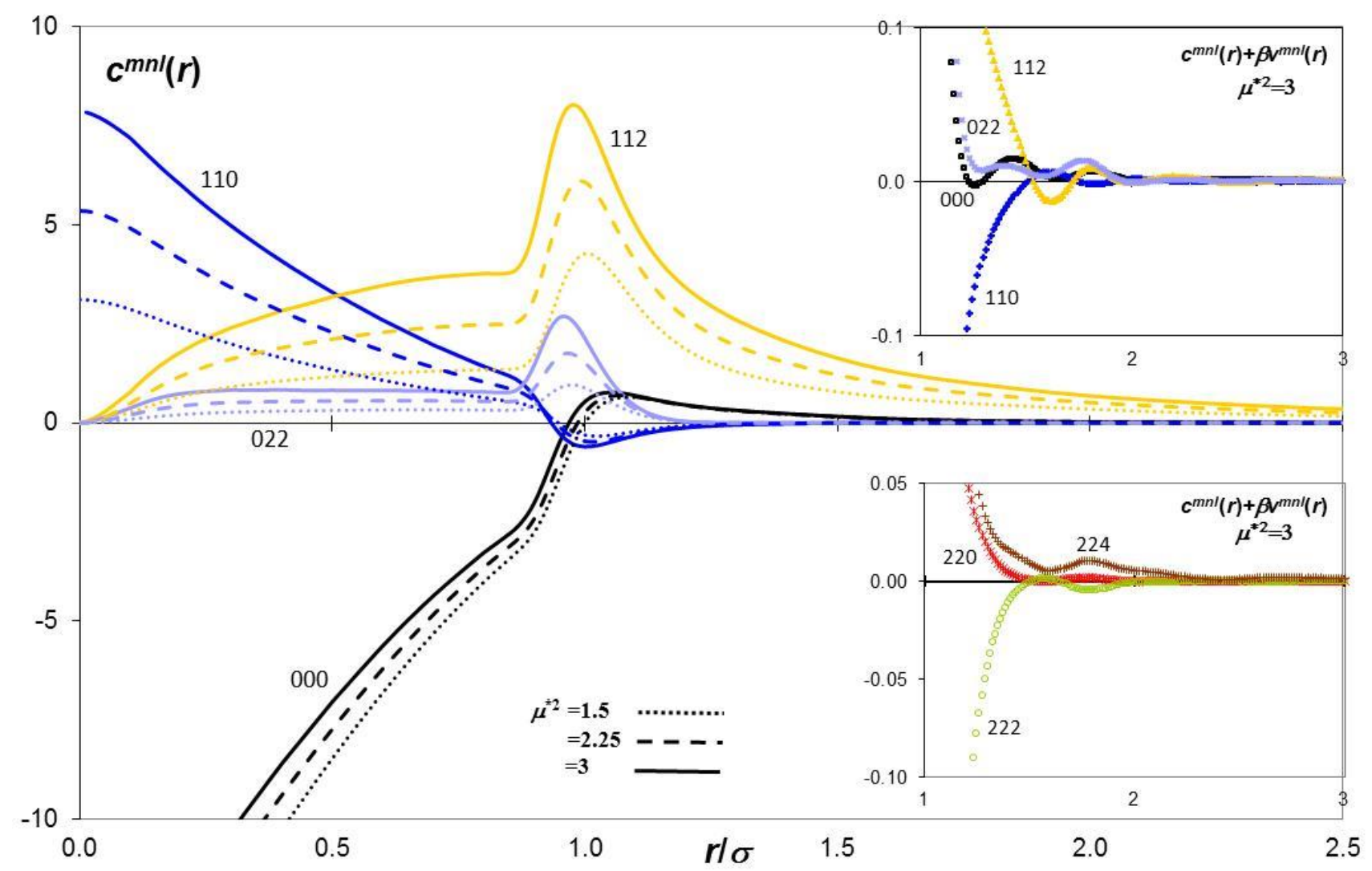


Figure 4

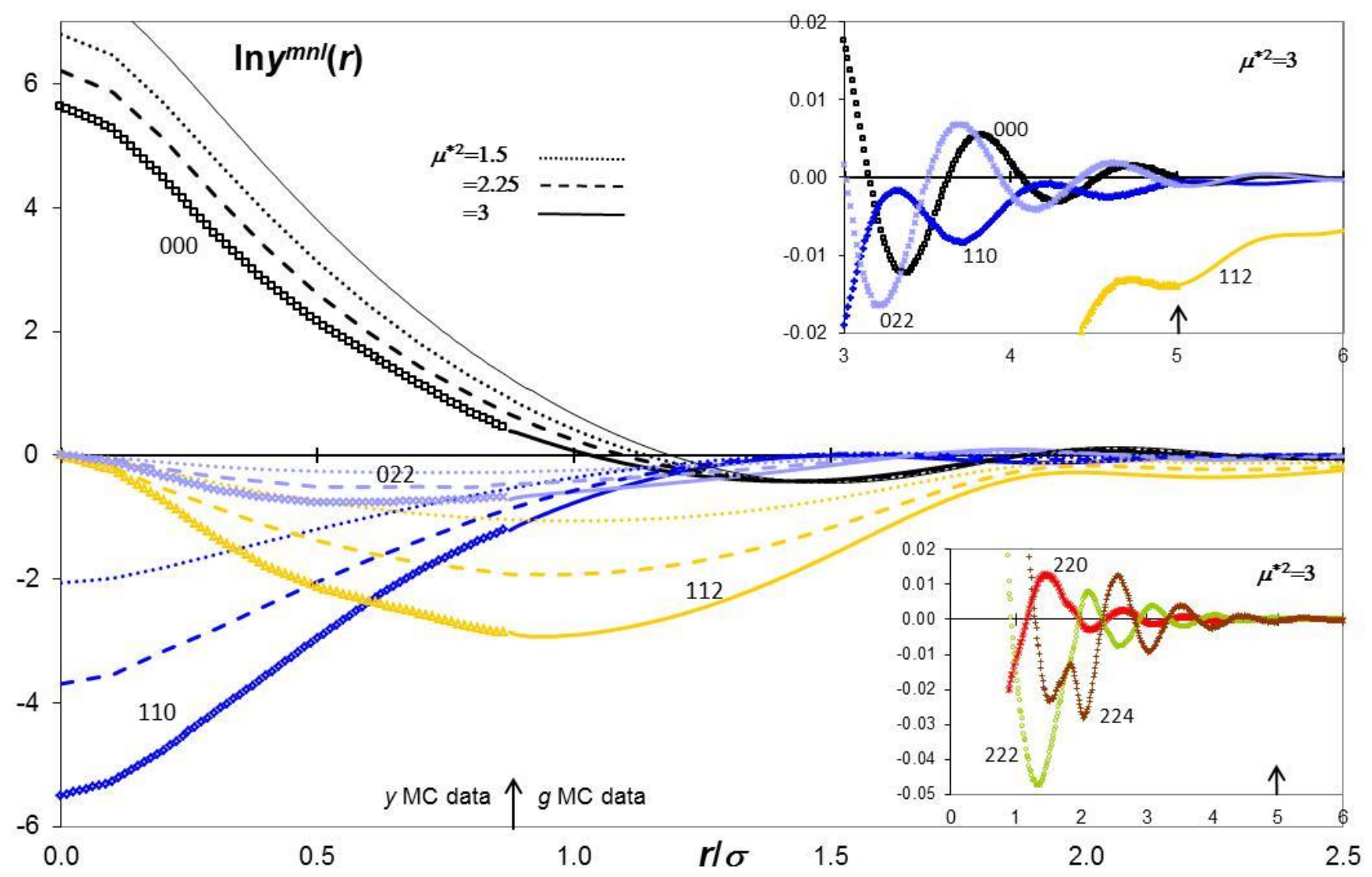


Figure 5

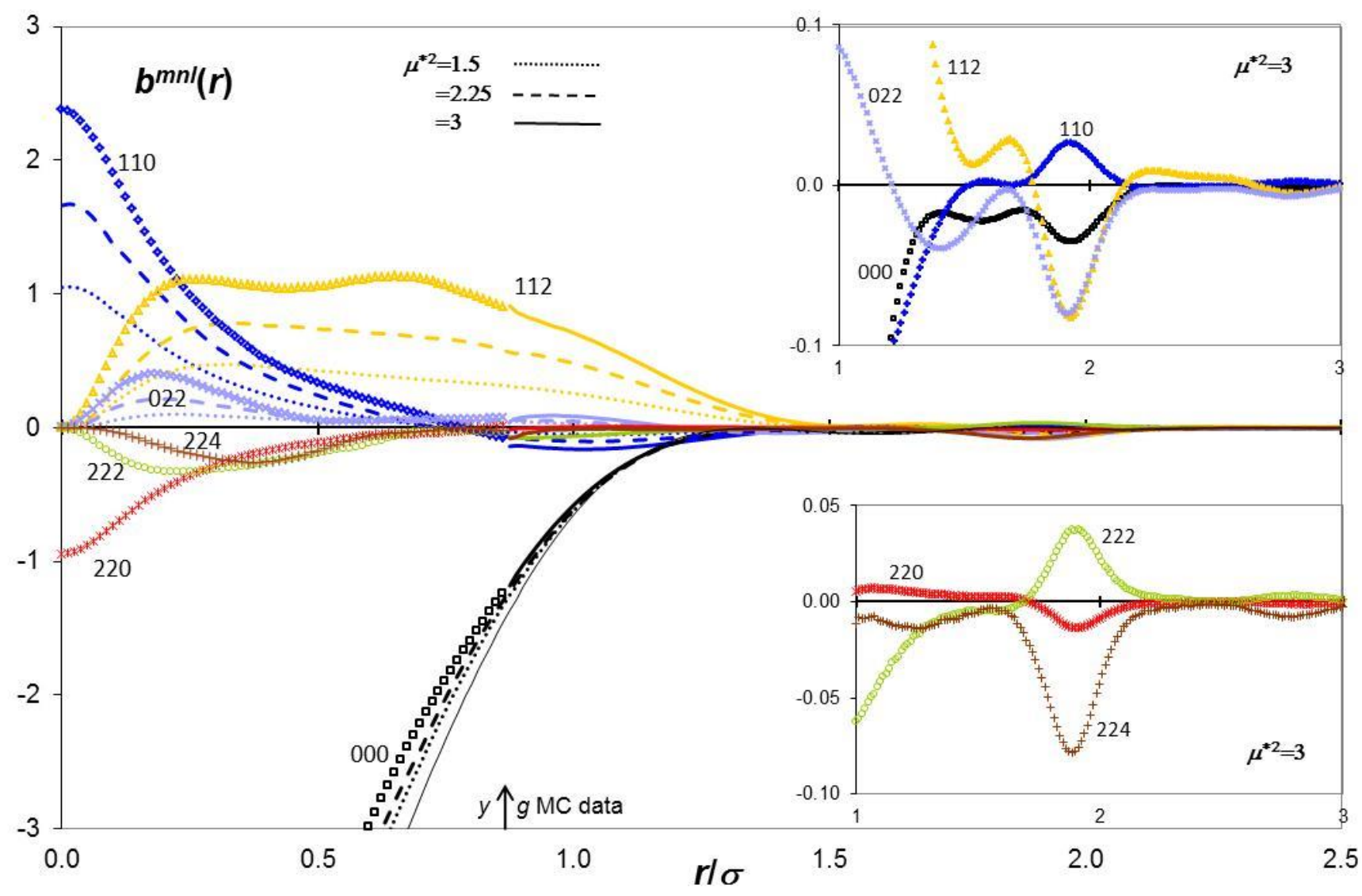


Figure 6

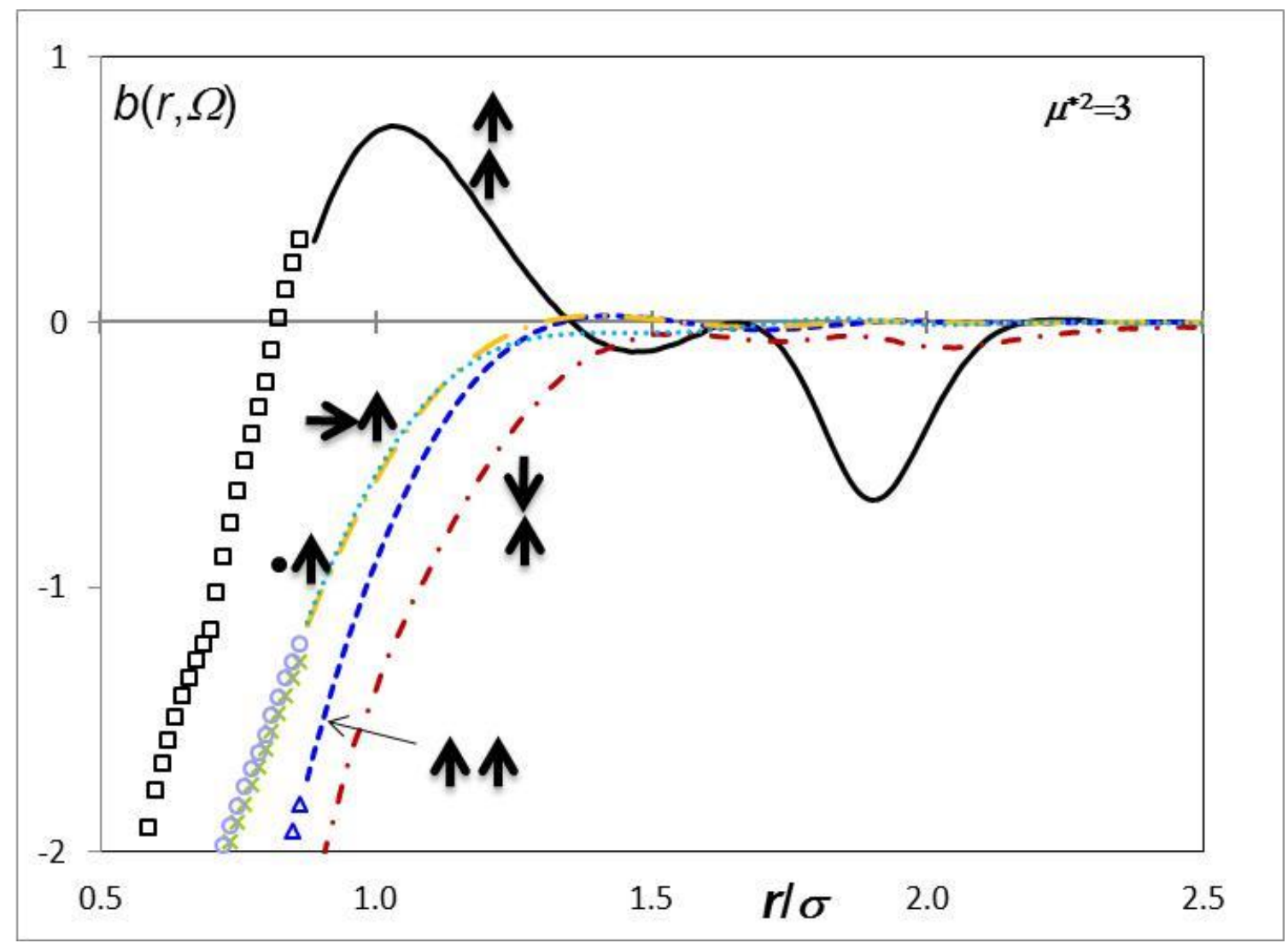


Figure 7

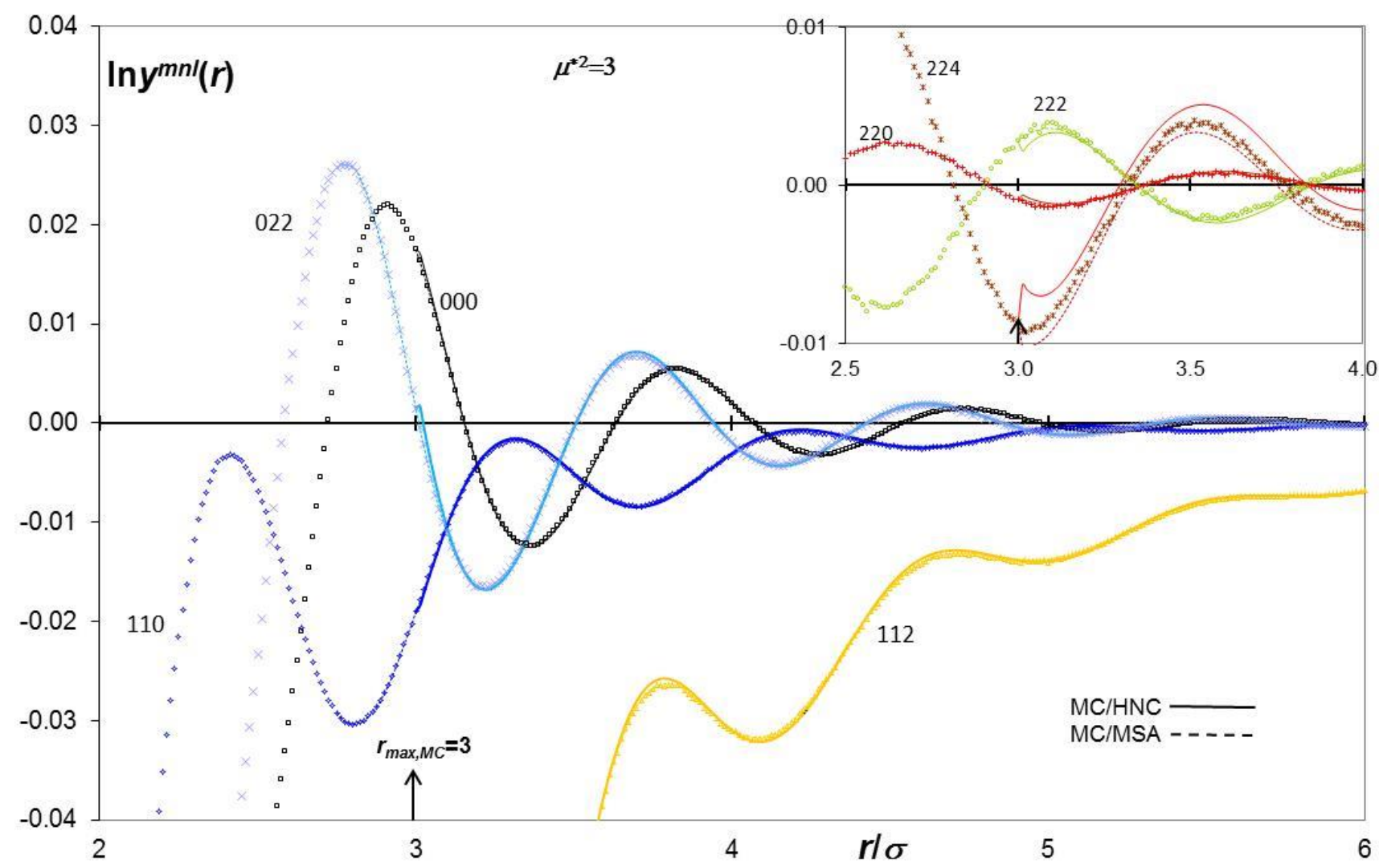


Figure 8

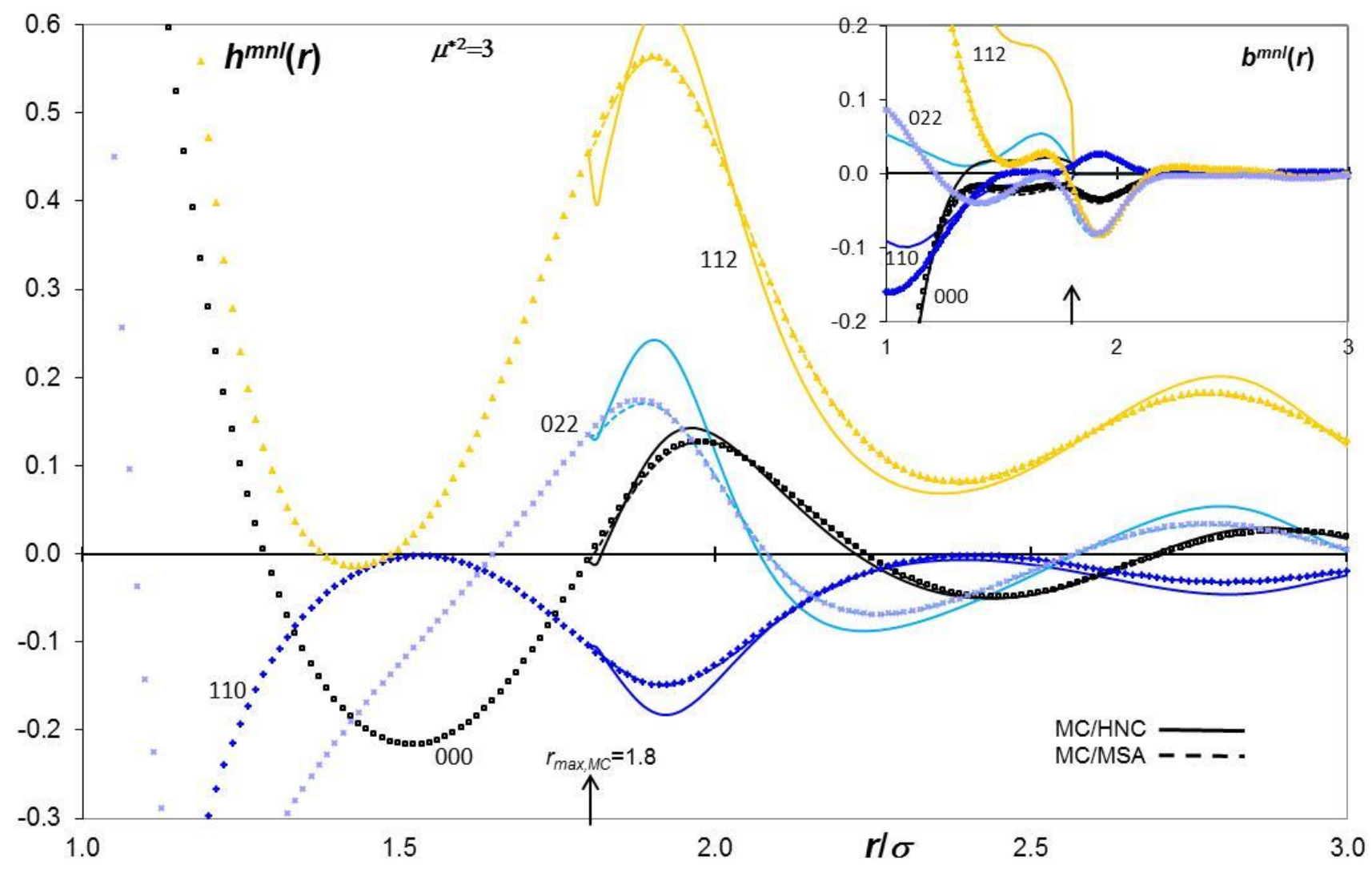


Figure 9

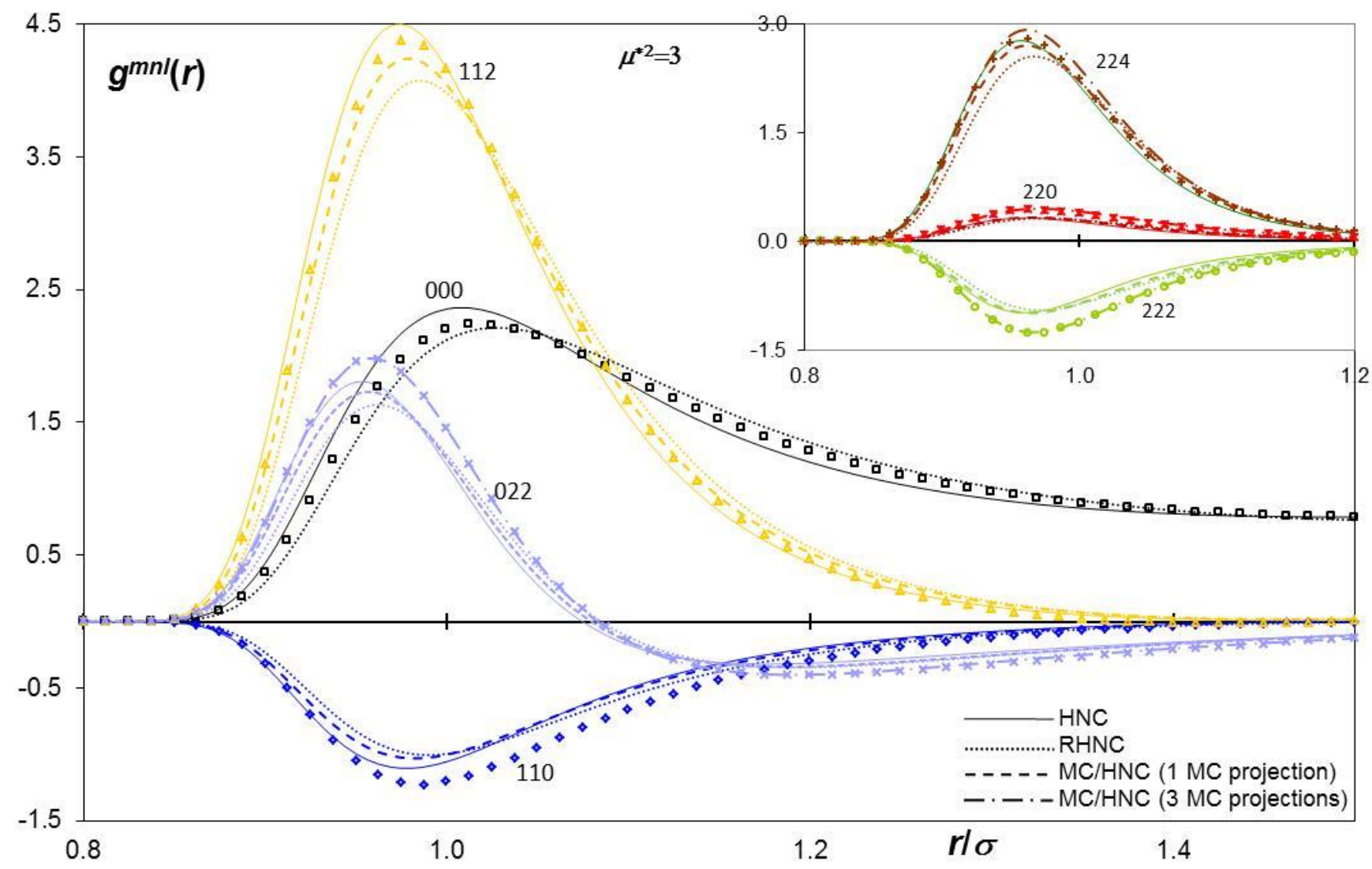


$\underline{\text { Figure } 10}$

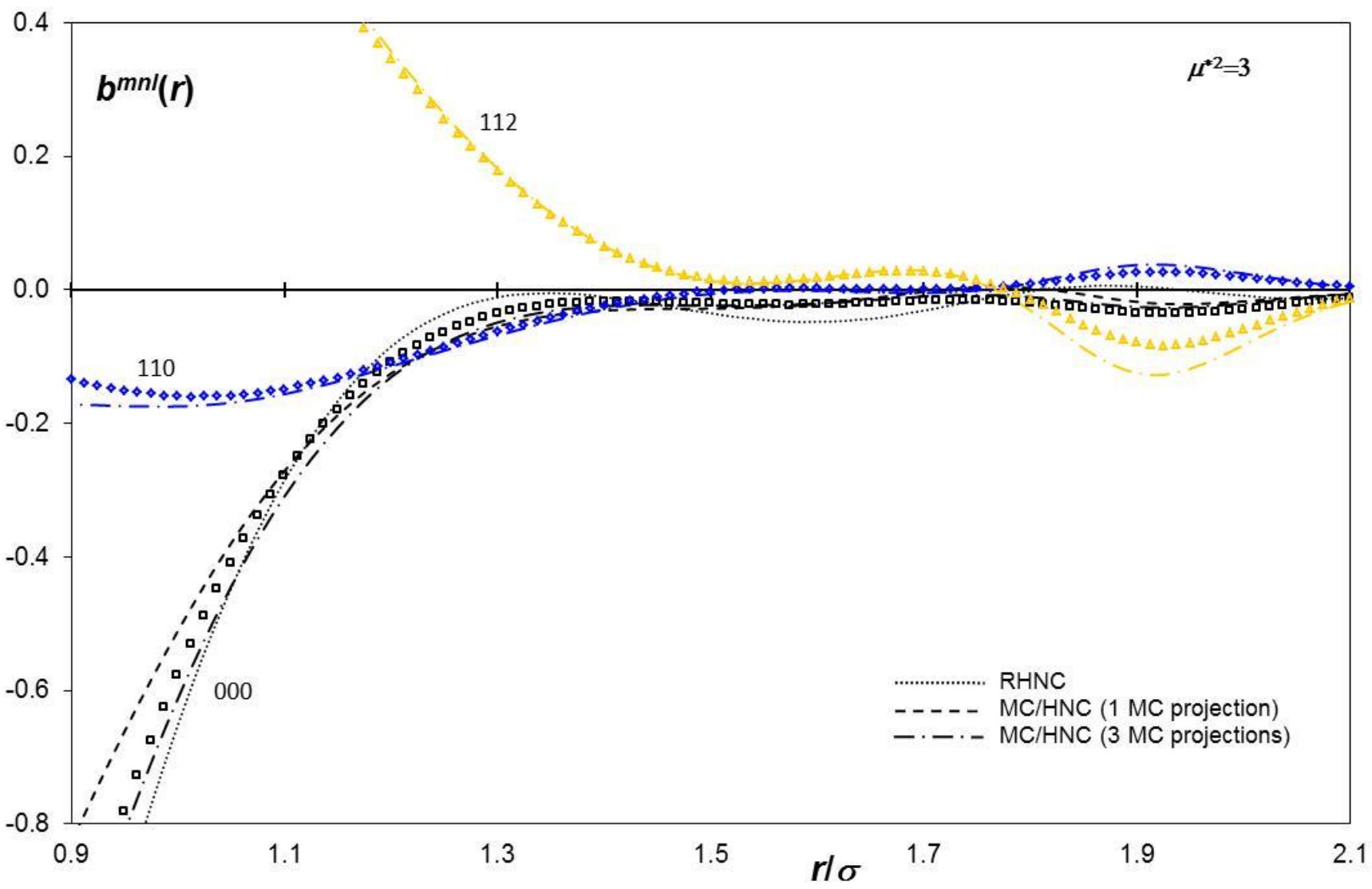

